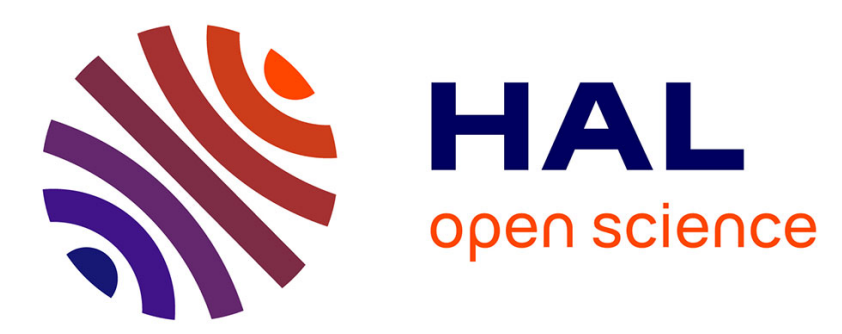

\title{
The discrete energy-momentum method. Conserving algorithms for nonlinear elastodynamics
}

\author{
J. C Simo, N. Tarnow
}

\section{To cite this version:}

J. C Simo, N. Tarnow. The discrete energy-momentum method. Conserving algorithms for nonlinear elastodynamics. Zeitschrift für Angewandte Mathematik und Physik, 1992, 43 (5), pp.757-792. 10.1007/BF00913408 . hal-01635171

\section{HAL Id: hal-01635171 \\ https://hal.science/hal-01635171}

Submitted on 14 Nov 2017

HAL is a multi-disciplinary open access archive for the deposit and dissemination of scientific research documents, whether they are published or not. The documents may come from teaching and research institutions in France or abroad, or from public or private research centers.
L'archive ouverte pluridisciplinaire HAL, est destinée au dépôt et à la diffusion de documents scientifiques de niveau recherche, publiés ou non, émanant des établissements d'enseignement et de recherche français ou étrangers, des laboratoires publics ou privés. 


\section{The discrete energy-momentum method. Conserving algorithms for nonlinear elastodynamics ${ }^{1}$}

By J. C. Simo and N. Tarnow, ${ }^{2}$ Div. of Applied Mechanics, Dept. of Mechanical Engineering, Stanford University, Stanford, CA 94305, U.S.A.

Dedicated to K. Kirchgässner on the occasion of his 60th birthday

\section{§1. Introduction and motivation}

Classical nonlinear continuum mechanics is built on the balance laws of linear and angular momentum, supplemented by balance of energy and a statement of the second law. These two additional laws are satisfied if the internal dissipation in the material $\mathscr{D}_{t}^{\text {int }}$, understood as the difference between the stress power and the rate of change of the internal energy, is positive. ${ }^{3}$ In particular, for nonlinear elastodynamics with conservative loading, the internal dissipation vanishes for smooth solutions and the total energy in the system $E_{t}^{\text {tot }}$ is exactly conserved. For non-smooth solutions; i.e. in the presence of shocks, dissipation arises and the energy in the system is no longer conserved; see Knowles [1979]. In what follows, we shall only be concerned with smooth solutions.

In the absence of external loading or in the presence of symmetries, the dynamics of a continuum exhibits two fundamental conservation laws in the pure traction initial boundary value problem (IBVP): The conservation laws of total linear momentum $\boldsymbol{L}_{t}$ and total angular momentum $\boldsymbol{J}_{t}$. In addition, the condition of positive dissipation yields an a-priori estimate on the rate of change of the total energy, which decays exactly by minus the dissipation. In summary:

$$
\frac{d \boldsymbol{L}_{t}}{d t}=\mathbf{0}, \quad \frac{d \boldsymbol{J}_{t}}{d t}=\mathbf{0} \quad \text { and } \quad \frac{d E_{t}^{\text {tot }}}{d t}=-\mathscr{D}_{t}^{\text {int }} \leq 0
$$

Invited Lecture, Presented at Oberwolfach January 1992.

Supported by AFOSR under Grant No. 2-DJA-826 with Stanford University.

3 Implicit here is the assumption that the entropy production due to heat conduction is positive; equivalently, the heat flux does not oppose the temperature gradient; see Truesdell and Noll [1972]. 
These fundamental properties of the continuum dynamics play a key role both in purely mathematical aspects of the IBVP as well as in many engineering applications. For instance, the realization of attitude controls for orbiting flexible satellites exploits these properties in a crucial manner.

Temporal and spatial finite difference/finite element discretizations of the continuum dynamics need not, and in general will not, inherit the conservation of momentum properties $(1.1)_{1,2}$ and the a-priori estimate $(1.1)_{3}$. The central goal of this work is the systematic construction of algorithmic approximations to the continuum dynamics that will inherit, by design, these conservation properties and the a-priori estimate. There is a sizable body of literature concerned with the design of algorithms possessing these conservation properties; see e.g. Bayliss and Isaacson [1975] and Labudde and Greenspan [1976a,b]. It is often the case, however, that algorithms designed to preserve the a-priori energy estimate result in violation of momentum conservation and vice versa. For instance, the conservation form of the mid-point rule is an exact momentum conserving algorithm which does not conserve energy for autonomous Hamiltonian systems, except in the linear regime; see Simo, Tarnow and Wong [1992]. On the opposite side, the method of Hughes, Liu and Caughy [1978] yields an energy conserving algorithm which does not preserve angular momentum. For rigid body mechanics, on the other hand, Simo and Wong [1991] show that the conservation form of the mid-point rule in body coordinates, together with the exponential mapping in the rotation group, define an exact energy and momentum conserving algorithm.

For smooth solutions in nonlinear elastodynamics, the model problem of interest here, the algorithmic treatment proposed below achieves exact conservation of both energy and total linear and angular momentum. Remarkably, the scheme amounts to a simple modification of the conservation form of the mid-point rule, according to the following prescription:

Step 1. Write a mid-point rule approximation to the IBVP for nonlinear elastodynamics, written in conservation form, with the symmetric stress tensor left open. We show that this step defines a 6-parameter family of exact momentum conserving algorithms, regardless of the specific form of the constitutive equation.

Step 2. For elastodynamics define the convected symmetric stress tensor by evaluating the gradient of the stored energy function at a convex combination of the right Cauchy-Green tensors at the initial and final times of a typical time increment.

From a mechanical standpoint, Step 1 is equivalent to establishing balance of linear momentum (in conservation form) on the mid-point configuration defined as the average of the initial and final configurations in a time step. It is somewhat surprising that global balance of momentum is satisfied for 
any symmetric stress tensor. For the Saint Venant-Kirchhoff model Step 2 has this remarkably simple interpretation: Evaluate the elastic constitutive equation with the average Lagragian strain tensor and not with the Lagrangian strain tensor of the average configuration. We remark that convex combinations of the right Cauchy-Green tensor make geometric since positive definiteness is preserved and, more importantly, all the tensors involved live in the same (reference) configuration.

Although nonlinear elastodynamics is an infinite dimensional Hamiltonian system, in the sense described in Simo, Marsden and Krishnaprasad [1989], the algorithmic treatment proposed herein does not rely on this Hamiltonian structure and is also applicable to dissipative systems. In fact, in contrast with the conservation form of the mid-point rule, it can be shown that the energy-momentum algorithms described herein do not define a symplectic transformation in phase space. See Arnold [1988] for an explanation of this terminology and De Vogelaere [1956], Feng Kan [1986], Zhong and Marsden [1988], Lasagni [1988], Sanz-Serna [1988] and Simo, Tarnow and Wong [1992] for the algorithmic implications. Nevertheless, the property of exact energy conservation induces a natural notion of algorithmic stability not present in symplectic schemes such as the mid-point rule. In fact, as demonstrated by our numerical simulations, both the mid-point and trapezoidal rules may produce numerical solutions exhibiting blow-up for sufficiently large step-sizes, while the proposed scheme renders solutions that remain stable.

\section{§2. The continuum problem. Conservation laws}

We summarize below basic results on continuum mechanics needed for our subsequent algorithmic developments presented in Section 3. This methodology is by no means restricted to the (dissipationless) Hamiltonian case, as exemplified in the continuum setting by nonlinear elastodynamics. In fact, our construction of exact momentum preserving algorithms is independent of the specific form adopted by the constitutive equations.

\subsection{Lagrangian description. The weak-form of balance of momentum}

In what follows $\mathscr{B} \subset \mathbb{R}^{n_{\text {dim }}}$ will denote the reference placement occupied by a continuum body where $1 \leq n_{\mathrm{dim}} \leq 3$ is the spatial dimension. The set $\mathscr{B}$ is assumed open, bounded, with smooth boundary $\partial \mathscr{B}$. In agreement with a Lagrangian description of the motion material points are labeled throughout by their position $X \in \mathscr{B}$ in the reference placement. 


\subsubsection{Some basic notation}

Let the reference boundary $\partial \mathscr{B}$ be partitioned into disjoint subsets such that $\partial \mathscr{B}=\overline{\Gamma_{\varphi} \cup \Gamma_{\sigma}}$ with $\Gamma_{\varphi} \cap \Gamma_{\sigma}=\emptyset$. The (smooth) manifold $Q$ of admissible configurations is then defined by

$$
Q:=\left\{\boldsymbol{\varphi}: \mathscr{B} \rightarrow \mathbb{R}^{n_{\operatorname{dim}}} \mid J(\varphi):=\operatorname{det}[D \varphi]>0 \text { and }\left.\varphi\right|_{\Gamma_{\varphi}}=\bar{\varphi}\right\},
$$

where $\bar{\varphi}$ is specified data on $\Gamma_{\varphi}$. For elastodynamics one typically assumes that $Q \subset\left[H^{s}(\mathscr{B})\right]^{n_{\text {dim }}}$ with $s>n_{\text {dim }} / 2+1$. A motion in some time interval $\square$ is a $C^{1}$ map $t \in \mathbb{Q} \rightarrow \varphi_{t} \in Q$, with associated material velocity field $V_{t}=\dot{\varphi}_{t}$ and canonical momenta $\pi_{t}=\varrho_{0} V_{t}$, where the superposed dot denotes time differentiation and $\varrho_{0}: \mathscr{B} \rightarrow \mathbb{R}_{+}$is the reference density. Throughout our developments we shall denote by $S_{t}$ the symmetric Piola-Kirchhoff or convected stress tensor associated with the motion $\varphi_{t}$ and related to the Cauchy stress tensor $\sigma_{t}$ via the well-known relation

$$
S_{t}=J\left(\varphi_{t}\right) D \varphi_{t}^{-1}\left(\sigma_{t} \circ \varphi_{t}\right) D \varphi_{t}^{-T},
$$

known as the Piola-transformation. In terms of the convected stress tensor the prescribed traction boundary condition takes the form

$$
D \varphi_{t} S_{t} \boldsymbol{N}=\overline{\boldsymbol{t}}_{\boldsymbol{t}} \text { on } \Gamma_{\sigma} \times \mathbb{\mathbb { t }},
$$

where $N: \partial \mathscr{B} \rightarrow S^{2}$ is the unit normal field to the reference boundary $\partial \mathscr{B}$

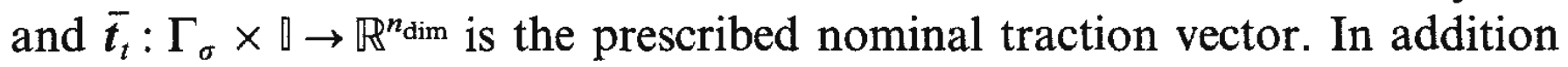
to the boundary tractions it will be assumed that the body is acted upon by a body force $f_{t}: \mathscr{B} \times \mathbb{Q} \rightarrow \mathbb{R}^{n_{\text {dim }}}$.

\subsubsection{The weak form of balance of momentum}

Let $\mathscr{V} \subset\left[H^{s}(\mathscr{B})\right]^{n_{\mathrm{dim}}}$ denote the space of test functions associated with admissible configurations in $Q$, defined in the usual fashion as

$$
\mathscr{V}:=\left\{\boldsymbol{\eta}: \mathscr{B} \rightarrow \mathbb{R}^{n_{\mathrm{dim}}}|\boldsymbol{\eta}|_{\Gamma_{\varphi}}=\mathbf{0}\right\} .
$$

Throughout our developments we denote by $\langle\cdot, \cdot\rangle$ the $L_{2}$-inner product on $\mathscr{B}$ of scalars, vectors or tensors, depending on the context. Setting $\langle\cdot, \cdot\rangle_{\Gamma}:=\int_{\Gamma_{\sigma}}(\cdot)(\cdot) d \Gamma$, the weak form of the balance of momentum at time $t \in \mathbb{\text { takes }}$ the form

$$
\left\langle\dot{\boldsymbol{\pi}}_{t}, \boldsymbol{\eta}\right\rangle+\left\langle D \boldsymbol{\varphi}_{t} \boldsymbol{S}_{t}, \operatorname{grad}[\boldsymbol{\eta}]\right\rangle=\left\langle\boldsymbol{f}_{t}, \boldsymbol{\eta}\right\rangle+\left\langle\overline{\boldsymbol{t}}_{t}, \boldsymbol{\eta}\right\rangle_{\Gamma} \quad \forall \boldsymbol{\eta} \in \mathscr{V}
$$

Here $\operatorname{grad}[\cdot]$ is the gradient operator of vector fields on $\mathscr{B}$ relative to a (material) coordinate system $\left\{X^{A}\right\}\left(A=1, \ldots, n_{\text {dim }}\right)$ on $\mathscr{B}$; i.e., in coordinates we have $(\operatorname{grad}[\eta])_{A}^{a}=\partial \eta^{a} / \partial X^{A}$ where $\eta^{a}\left(a=1, \ldots, n_{\mathrm{dim}}\right)$ are the components of $\eta \in \mathscr{V} . D \varphi_{t}$, on the other hand, denotes the derivative of 
point maps relative to material coordinates; i.e. $\left(D \varphi_{t}\right)_{A}^{a}=\partial \varphi^{a} / \partial X^{A}$. In $\mathbb{R}^{3}$, $D[\cdot]$ and $\operatorname{grad}[\cdot]$ can be identified. The variational equation (2.5a) is supplemented by the local relations

$$
V_{t}=\dot{\varphi}_{t} \quad \text { and } \quad \pi_{t}=\varrho_{0} V_{t} \quad \text { in } \overline{\mathscr{B}} \times \mathbb{Q} .
$$

along with the initial conditions

$$
\left.\varphi_{t}\right|_{t=0}=\varphi_{0} \text { and }\left.\pi_{t}\right|_{t=0}=\varrho_{0} V_{0} \quad \text { in } \mathscr{B} \text {. }
$$

Equations $(2.5 \mathrm{a}, \mathrm{b}, \mathrm{c})$ define a nonlinear initial boundary value problem for the motion $t \in \mathbb{V} \mapsto \varphi_{t} \in Q$ of the continuum system once a constitutive equation relating the convected stress tensor $S_{t}$ to the motion $\varphi_{t}$ is specified.

\subsection{Constitutive equations. Positive internal dissipation}

As pointed out above, the algorithmic approach described below encompasses a general class of constitutive relations, not necessarily restricted to elastodynamics, subject to the only requirement of positive dissipation. This restriction leads naturally to meaningful definitions of nonlinear algorithmic stability.

\subsubsection{Energy balance: Positive dissipation}

Let $K$ and $\mathscr{P}_{t}^{\text {int }}$ denote the kinetic energy and the stress power of the continuum system, respectively, as defined by the relations

$$
K_{t}=\frac{1}{2}\left\langle\pi_{t}, V_{t}\right\rangle=\frac{1}{2}\left\|\varrho_{0} V_{t}\right\|^{2} \text { and } \mathscr{P}_{t}^{\text {int }}=\left\langle D \varphi_{t} S_{t}, \operatorname{grad}\left[\boldsymbol{V}_{t}\right]\right\rangle \text {. }
$$

We further denote by $\mathscr{P}_{t}^{\text {ext }}$ the expended power of the external loading, defined by the expression

$$
\mathscr{P}_{t}^{\text {ext }}:=\left\langle\boldsymbol{f}_{t}, \boldsymbol{V}_{t}\right\rangle+\left\langle\overline{\boldsymbol{t}}_{t}, \boldsymbol{V}_{t}\right\rangle_{\Gamma} \quad t \in \mathbb{Q} .
$$

For fixed time $t \in \mathbb{\|}$ the material velocity field defines an admissible test function. Therefore, setting $\boldsymbol{\eta}=\boldsymbol{V}_{t}$ in (2.5) and making use of the notations above yields the result

$$
\frac{d K_{t}}{d t}+\mathscr{P}_{t}^{\text {int }}=\mathscr{P}_{t}^{\text {ext }}, \quad \text { for any } t \in \mathbb{V},
$$

known as the theorem of expended power.

Now let $E_{t}^{\text {int }}:=\int_{\mathscr{B}} e d \mathscr{B}$ be the internal energy function which specifies the stored energy in the continuum system and define the internal dissipation function $\mathscr{D}_{t}^{\text {int }}$ of the system as the difference between the total stress power $\mathscr{P}_{t}^{\text {int }}$ and the rate of change of the internal energy. We restrict our attention to constitutive equations obeying the following reduced dissipation inequality

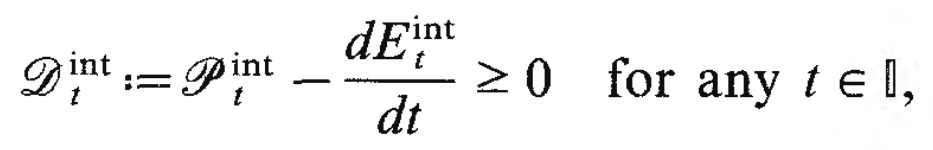


which (in the absence of heat conduction) can be viewed as an equivalent statement of the Clausius-Duhem form of the second law. Combining (2.8) and (2.9) yields the following result for the rate of change of the kinetic and internal energies:

$$
\frac{d E_{t}^{\text {tot }}}{d t}=\mathscr{P}_{t}^{\text {ext }}-\mathscr{D}_{t}^{\text {int }} \quad \text { with } \quad E_{t}^{\text {tot }}:=K_{t}+E_{t}^{\text {int }} \text { and } \mathscr{D}_{t}^{\text {int }} \geq 0 \text { for } t \in \mathbb{Q}
$$

This relation plays a crucial role in our subsequent algorithmic developments.

\subsubsection{Constitutive equations: Examples}

Two representative and fairly classical examples which fall within the preceding framework are:

i. Nonlinear elastodynamics. Here the internal energy is defined locally in terms of the deformation gradient $D \varphi_{t}$ of the motion by means of the frame-invariant stored energy function $e=\hat{e}\left(D \varphi_{t}^{T} D \varphi_{t}\right)$. The convected stress tensor $\boldsymbol{S}_{t}$ and the internal dissipation then become

$$
S_{t}=2 \nabla \hat{e}\left(D \varphi_{t}^{T} D \varphi_{t}\right) \quad \text { and } \quad \mathscr{D}_{t}^{\text {int }} \equiv 0 \quad \forall t \in \mathbb{0} .
$$

The Cauchy stress tensor $\sigma_{t}$ is obtained from $(2.11)_{1}$ via the Piola-transformation (2.2). The fact that $\mathscr{D}_{t}^{\text {int }}$ vanishes for an elastic material justifies the denomination 'internal dissipation' assigned to $\mathscr{D}_{t}^{\text {int }}$. For the incompressible problem, obtained by appending the constraint $J\left(\varphi_{t}\right)=1$ in $\Omega \times \mathbb{Q}$, the Cauchy stress tensor is defined up to a spherical part $p \mathbf{1}$ involving the hydrostatic pressure $p$. Nonlinear elastodynamics possesses a well-understood Hamiltonian structure; see Simo, Marsden and Krishnaprasad [1988] for detailed exposition.

ii. Incompressible Navier-Stokes system. Let $\boldsymbol{v}_{t}:=\boldsymbol{V}_{t} \circ \boldsymbol{\varphi}_{t}^{-1}$ be the spatial velocity field with spatial gradient $\nabla \boldsymbol{v}_{t}=\left(\operatorname{grad}\left[\boldsymbol{V}_{t}\right] D \boldsymbol{\varphi}_{t}^{-1}\right) \circ \boldsymbol{\varphi}_{t}^{-1}$. In the linear incompressible viscous model the Cauchy stress is defined by the constitutive relation

$$
\boldsymbol{\sigma}_{t}=-p \mathbf{1}+2 \mu \operatorname{sym}\left[\nabla \boldsymbol{v}_{t}\right] \quad \text { with } \quad J\left(\varphi_{t}\right)=1 \Leftrightarrow \operatorname{div}\left[\boldsymbol{v}_{t}\right]=0 \quad \forall t \in \mathbb{U} .
$$

The convected stress tensor $S_{t}$ is obtained from (2.12) via the Piola-transformation (2.2). The internal dissipation function is

$$
\mathscr{D}_{t}^{\text {int }}=\mu \int_{\varphi_{t}(\mathscr{B})} \nabla \boldsymbol{v}_{t} \cdot \nabla \boldsymbol{v}_{t} d x \geq 0 \text { for } \mu \geq 0,
$$

while the internal energy vanishes identically; i.e., $E_{t}^{\text {int }} \equiv 0$. 
Several classical models in nonlinear solid mechanics exhibit positive dissipation while retaining a non-vanishing stored energy function; i.e., finite plasticity.

\subsection{The unforced problem: Balance laws and a-priori estimate}

A number of important applications involve the solution of the initial boundary value problem with prescribed zero loading and driven, therefore, solely by initial conditions; i.e., the unforced problem. This situation is also relevant to the nonlinear stability analysis of algorithmic approximations (see Simo [1991]) and will be considered in what follows.

\subsubsection{Conservation laws and a-priori estimate}

Recall that the resultant force $\boldsymbol{F}_{t}^{\text {ext }}$ and the resultant moment $\boldsymbol{T}_{t}^{\text {ext }}$ of the prescribed external loading (relative to some inertial frame) are defined by the expressions

$$
\boldsymbol{F}_{t}^{\mathrm{ext}}:=\int_{\mathscr{B}} \boldsymbol{f}_{t} d \mathscr{B}+\int_{\Gamma_{\sigma}} \overline{\bar{t}_{t}} d \Gamma \quad \text { and } \quad \boldsymbol{T}_{t}^{\mathrm{ext}}:=\int_{\mathscr{B}} \boldsymbol{\varphi}_{t} \times \boldsymbol{f}_{t} d \mathscr{B}+\int_{\Gamma_{\sigma}} \boldsymbol{\varphi}_{t} \times \overline{\boldsymbol{t}_{t}} d \Gamma \text {. }
$$

The following assumptions specify the framework relevant to the analysis of algorithms designed to inherit conservation laws present in the continuum problem:

(A1) Assume that $\boldsymbol{F}_{t}^{\text {ext }}=\mathbf{0}, \boldsymbol{T}_{t}^{\text {ext }}=\mathbf{0}$ and $\mathscr{P}_{t}^{\text {ext }}=0, \forall t \in \mathbb{Q}$.

(A2) Consider pure Neumann data; equivalently, assume $\Gamma_{\varphi}=\emptyset$.

From a physical standpoint these two conditions can be realized by considering the motion of a continuum body subject to no boundary restrictions, with prescribed zero loading, driven by specified initial conditions. If $\boldsymbol{L}_{t}$ and $J_{t}$ denote the total linear and total angular momentum defined by the standard expressions

$$
\boldsymbol{L}_{t}:=\int_{\mathscr{B}} \pi_{t} d \mathscr{B} \quad \text { and } \quad \boldsymbol{J}_{t}:=\int_{\mathscr{B}} \boldsymbol{\varphi}_{t} \times \pi_{t} d \mathscr{B},
$$

under assumptions (A1) and (A2) above the ensuing motion possesses two fundamental conservation laws and one a-priori estimate:

$$
\frac{d \boldsymbol{L}_{t}}{d t}=\mathbf{0}, \quad \frac{d \boldsymbol{J}_{t}}{d t}=\mathbf{0} \quad \text { and } \quad \frac{d E_{t}^{\text {tot }}}{d t}=-\mathscr{D}_{t}^{\text {int }} \leq 0 \quad \forall t \in \mathbb{Q} .
$$

The a-priori estimate $(2.16)_{3}$ follows at once from relation (2.10) since $\mathscr{P}_{t}^{\text {ext }}=0$. Relations $(2.16)_{1,2}$ are the familiar conservation laws of total linear 
and total angular momentum, respectively. These latter two results can be derived directly from the weak form (2.5) of the momentum equation by the argument given below. The same approach is exploited in the algorithmic analysis of the momentum conserving properties presented in Section 3.2.

\subsubsection{Conservation of momentum and the weak form}

Since $\Gamma_{\varphi}=\emptyset$ by assumption, it follows that test functions in $\mathscr{V}$ are unrestricted on the boundary. In particular, two specific class of test functions are now contained in $\mathscr{V}$ :

i. Infinitesimal translations, defined by $\boldsymbol{\eta}(\boldsymbol{X})=\boldsymbol{\xi}, \forall X \in \overline{\mathscr{B}}$, where $\xi \in \mathbb{R}^{n_{\mathrm{dim}}}$ is a fixed vector. For this class of test functions we have $\operatorname{grad}[\boldsymbol{\eta}] \equiv \mathbf{0}$. Expression (2.5) along with definitions $(2.14)_{1}$ and $(2.15)_{1}$ give

$$
0=\left\langle\boldsymbol{f}_{t}, \boldsymbol{\xi}\right\rangle+\left\langle\overline{\boldsymbol{t}}_{t}, \boldsymbol{\xi}\right\rangle_{\Gamma}-\left\langle\dot{\boldsymbol{\pi}}_{t}, \boldsymbol{\xi}\right\rangle=\left[\boldsymbol{F}_{t}^{\mathrm{ext}}-\dot{\boldsymbol{L}}_{t}\right] \cdot \boldsymbol{\xi},
$$

which implies $\boldsymbol{F}_{t}^{\text {ext }}-\dot{\boldsymbol{L}}_{t}=\mathbf{0}$ since (2.17) holds for any $\xi \in \mathbb{R}^{3}$ and collapses to $(2.16)_{1}$ since $\boldsymbol{F}_{t}^{\mathrm{ext}}=\mathbf{0}$.

ii. Infinitesimal rotations $\left(n_{\mathrm{dim}}=3\right.$ ), defined by $\boldsymbol{\eta}=\boldsymbol{\xi} \times \boldsymbol{\varphi}_{t}$ where $\boldsymbol{\xi} \in \mathbb{R}^{3}$ is a fixed vector defining the rotation axis. Following a standard convention let $\hat{\xi}$ denote the associated skew-symmetric matrix defined by the relation $\hat{\xi} a:=\xi \times a$ for any vector $a \in \mathbb{R}^{3}$. For this class of test functions a direct calculation gives $\operatorname{grad}[\eta]=\hat{\xi} D \varphi_{t}$. Inserting this result in (2.5) and using $(2.14)_{2}$ along with $(2.15)_{2}$ yields, after a straightforward manipulation, the result

$$
\begin{aligned}
\left\langle D \varphi_{t} S_{t}, \hat{\xi} D \varphi_{t}\right\rangle & =\left\langle\varphi_{t} \times f_{t}, \xi\right\rangle+\left\langle\varphi_{t} \times \overline{\boldsymbol{t}}_{t}, \boldsymbol{\xi}\right\rangle_{\Gamma}-\left\langle\boldsymbol{\varphi}_{t} \times \dot{\pi}_{t}, \boldsymbol{\xi}\right\rangle \\
& =\left[\boldsymbol{T}_{t}^{\mathrm{ext}}-\dot{\boldsymbol{J}}_{t}\right] \cdot \boldsymbol{\xi} .
\end{aligned}
$$

Since the Cauchy stress tensor $\sigma_{t}$ is symmetric, from the Piola transformation (2.2) we conclude that $D \varphi_{t} S_{t} D \varphi_{i}^{T}$ is also symmetric and, therefore,

$$
\left\langle D \varphi_{t} S_{t}, \hat{\xi} D \varphi_{t}\right\rangle=\left\langle D \varphi_{t} S_{t} D \varphi_{t}^{T}, \hat{\xi}\right\rangle \equiv 0 \quad \text { since } \hat{\xi}=-\hat{\xi}^{T} .
$$

Since $\xi \in \mathbb{R}^{3}$ is arbitrary combining (2.19) and (2.18) yields $\boldsymbol{T}_{t}^{\text {ext }}-\dot{\boldsymbol{J}}=\mathbf{0}$ which implies $(2.16)_{1}$ since $\boldsymbol{T}_{t}^{\text {ext }}=\mathbf{0}$.

\section{§3. Time discretization. Conserving schemes}

The dynamics generated by a number of important continuum system, including nonlinear elastodynamics, finite plasticity and the Navier-Stokes system among many others, obeys the conservation laws $(2.16)_{1,2}$ and a-priori estimate $(2.16)_{3}$ provided assumptions (A1) and (A2) hold. Our 
main goal in this Section is the design of algorithmic approximations which will inherit these crucial properties of the continuum dynamics.

\subsection{The algorithmic framework. Conserving schemes}

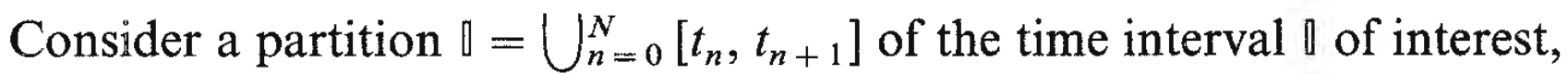
focus attention on a typical time interval $\left[t_{n}, t_{n+1}\right]$ and let $\Delta t:=t_{n+1}-t_{n}>0$ denote the corresponding time-step size. Following a standard convention, we shall denote by either $(\cdot)_{n}$ or $(\cdot)_{n+1}$ the algorithmic approximations at times $t_{n}$ and $t_{n+1}$ to the continuum (time-dependent) variable $(\cdot)_{t}$. In our algorithmic analysis we use the following restatement of condition (A1) of Section 2

$\left(\mathrm{A} 1^{*}\right)$ For the unforced problem, assume that $\boldsymbol{F}_{t}^{\text {ext }}=\mathbf{0}, \boldsymbol{T}_{t}^{\text {ext }}=\mathbf{0}$ and $\mathscr{P}_{t}^{\text {ext }}=0$, for all $t \in\left[t_{n}, t_{n+1}\right]$.

We assume all the variables of interest specified at time $t_{n}$; in particular, the initial data $\left(\varphi_{n}, \pi_{n}\right)$ and the symmetric Piola-Kirchhoff stress : $(X)$, for all $X \in \overline{\mathscr{B}}$. Our goal is the design of a consistent approximation to the IBVP defined by $(2.5 \mathrm{a}, \mathrm{b}, \mathrm{c})$, along with a suitable constitutive equation of the type described in Section 2.2, possessing the following properties whenever assumptions $(\mathrm{A} 1 *)$ and $(\mathrm{A} 2)$ hold:

i. Discrete momentum conservation in the sense that

$$
\boldsymbol{L}_{n+1}=\boldsymbol{L}_{n} \quad \text { and } \quad \boldsymbol{J}_{n+1}=\boldsymbol{J}_{n}, \quad n=0,1, \ldots, N .
$$

ii. Discrete energy conservation, in the sense that

$$
E_{n+1}^{\text {tot }} \leq E_{n}^{\text {tot }} \text { and } \quad E_{n+1}^{\text {tot }} \equiv E_{n}^{\text {tot }} \Leftrightarrow \mathscr{D}_{t}^{\text {int }} \equiv 0, \quad n=0,1, \ldots, N
$$

The requirement $E_{n+1}^{\text {tot }} \equiv E_{n}^{\text {tot }}$ whenever $\mathscr{D}_{t}^{\text {int }} \equiv 0$ in $\llbracket$ corresponds to exact energy conservation in the Hamiltonian case and will be the only situation addressed below within the specific context of elastodynamics. Algorithmic approximations possessing the properties in (3.1) will be referred to as energy-momentum conserving schemes in what follows.

For nonlinear elastodynamics, the design of a conserving scheme is accomplished in two steps. First, we identify a class of one-step implicit algorithms which, remarkably, achieve exact momentum conservation with total independence of the approximation used for the convected stress tensor. In fact, the property of exact momentum conservation holds for any constitutive model. Second, we specialize this class of algorithms to nonlin- 
ear elasticity and show that an approximation to the constitutive equation $(2.11)_{1}$ can always be constructed such that exact conservation of energy is achieved. From a geometric standpoint this two-step construction is analogous to the approach recently advocated in Simo, Tarnow and Wong [1992]. The actual definition of the two steps, however, is entirely different.

\subsection{A class of exact momentum conserving algorithms}

Consider a one-parameter family of configurations $\varphi_{n+\alpha} \in Q$, with associated velocity field $V_{n+\alpha}$, defined by the convex combination

$$
\left.\begin{array}{l}
\varphi_{n+\alpha}=\alpha \varphi_{n+1}+(1-\alpha) \varphi_{n} \\
V_{n+\alpha}=\alpha V_{n+1}+(1-\alpha) V_{n}
\end{array}\right\}, \quad \alpha \in[0,1] .
$$

Thus, associated with the reference placement $\mathscr{B}$ of the continuum body we have the placements $\varphi_{n}(\mathscr{B}), \varphi_{n+\alpha}(\mathscr{B})$ and $\varphi_{n+1}(\mathscr{B})$ illustrated in Figure 3.1. The corresponding convected stress tensors are denoted by $\boldsymbol{S}_{n}, \boldsymbol{S}_{n+\alpha}$ and $S_{n+1}$, respectively. With the preceding notation in hand now consider the following algorithmic approximation to the weak form (2.5a) of

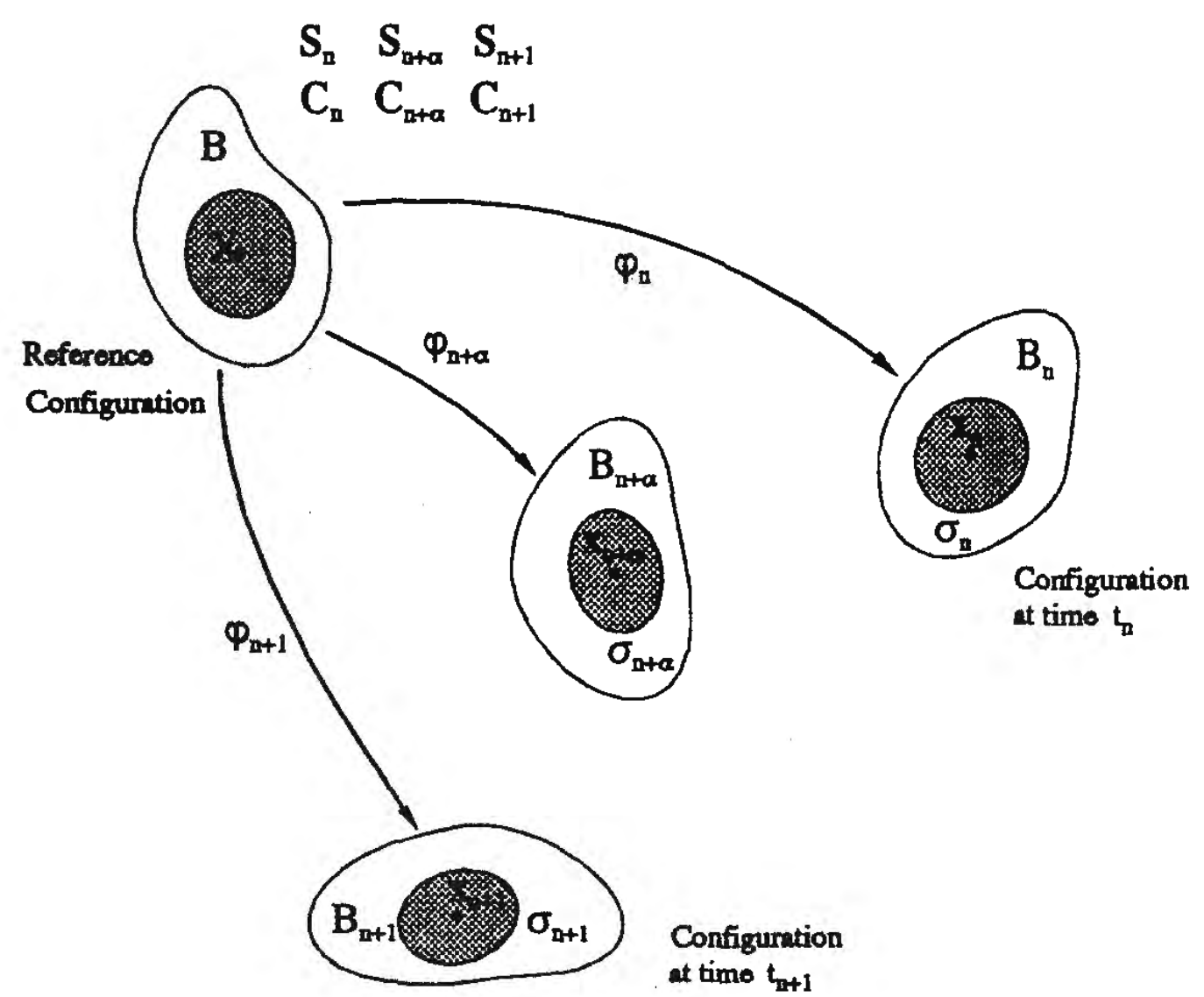

Figure 3.1

Illustration of the three configurations involved in the formulation of the algorithm within the step $\left[t_{n}, t_{n+1}\right]$. Note that all the convected stress and right Cauchy-Green tensors live in the reference configuration $\mathscr{B}$. 
balance of momentum

$$
\begin{gathered}
\frac{1}{\Delta t}\left\langle\boldsymbol{\pi}_{n+1}-\boldsymbol{\pi}_{n}, \boldsymbol{\eta}\right\rangle+\left\langle D \boldsymbol{\varphi}_{n+\alpha} \boldsymbol{S}, \operatorname{grad}[\boldsymbol{\eta}]\right\rangle \\
=\left\langle\boldsymbol{f}_{n+\alpha}, \boldsymbol{\eta}\right\rangle+\left\langle\overline{\boldsymbol{t}}_{n+\alpha}, \boldsymbol{\eta}\right\rangle_{\Gamma} \quad \forall \boldsymbol{\eta} \in \mathscr{V},
\end{gathered}
$$

where $\boldsymbol{S}=\boldsymbol{S}^{T}$ is a completely arbitrary symmetric tensor field defined on $\mathscr{B}$. The algorithmic weak form (3.3a) is supplemented by the local relations

$$
\frac{1}{\Delta t}\left[\varphi_{n+1}-\varphi_{n}\right]=V_{n+\alpha} \text { and } \pi_{n+\alpha}:=\varrho_{0} V_{n+\alpha}
$$

which complete the algorithmic approximation to equations $(2.5 \mathrm{a}, \mathrm{b})$. We emphasize that the symmetric tensor $\boldsymbol{S}$ is regarded here as defining a 6-parameter family of algorithms to be further specialized below. Our objective is to examine the conservation properties inherited by the time discretization $(2.5 \mathrm{a}, \mathrm{b})$.

\subsubsection{Discrete momentum conservation}

Let $\boldsymbol{F}_{n+\alpha}^{\text {ext }}$ and $\boldsymbol{T}_{n+\alpha}^{\text {ext }}$ denote the total resultant force and resultant moment defined by (2.14) and evaluated at time $t_{n+1}$. We show below that if $\boldsymbol{S}$ is symmetric but otherwise completely arbitrary the algorithmic approximation defined by $(3.3 \mathrm{a}, \mathrm{b})$ yields the following relations

$$
\left.\begin{array}{ll}
\boldsymbol{L}_{n+1}-\boldsymbol{L}_{n}=\Delta t \boldsymbol{F}_{n+\alpha}^{\mathrm{ext}} & \text { for any } \alpha \in[0,1], \\
\boldsymbol{J}_{n+1}-\boldsymbol{J}_{n}=\Delta t \boldsymbol{T}_{n+\alpha}^{\text {ext }} & \text { only if } \alpha=\frac{1}{2} .
\end{array}\right\}
$$

In particular, since $\boldsymbol{F}_{n+\alpha}^{\mathrm{ext}}=\mathbf{0}$ and $\boldsymbol{T}_{n+\alpha}^{\mathrm{ext}}=\mathbf{0}$ if conditions (A1*) and (A2) hold, relations (3.4) yield the discrete momentum conservation law (3.1) within the interval $\left[t_{n}, t_{n+1}\right]$ provided that $\alpha=\frac{1}{2}$.

Relation (3.4) follows immediately from (3.3a) merely by setting $\boldsymbol{\eta}=\boldsymbol{\xi}$ (constant) in $\mathscr{B}$ and noting that $\operatorname{grad}[\xi]=\mathbf{0}$. To prove relation $(3.4)_{2}$ we take $\xi \in \mathbb{R}^{3}$ and use definition (3.2) to compute

$$
\begin{aligned}
\xi \cdot\left(J_{n+1}-J_{n}\right)= & \left\langle\xi, \varphi_{n+1} \times \pi_{n+1}-\varphi_{n} \times \pi_{n}\right\rangle \\
= & \left.\left\langle\xi, \varphi_{n+1} \times\left(\pi_{n+1}-\pi_{n}\right)+\left(\varphi_{n+1}-\varphi_{n}\right) \times \pi_{n}\right)\right\rangle \\
= & \left\langle\xi, \varphi_{n+\alpha} \times\left(\pi_{n+1}-\pi_{n}\right)\right\rangle \\
& +\left\langle\xi,\left(\varphi_{n+1}-\varphi_{n}\right) \times\left(\alpha \pi_{n}+(1-\alpha) \pi_{n+1}\right)\right\rangle .
\end{aligned}
$$

The first term of the last right hand side of (3.5) can be evaluated as follows. We observe that $\boldsymbol{\eta}=\boldsymbol{\xi} \times \boldsymbol{\varphi}_{n+\alpha}$ is an admissible test function in $\mathscr{V}$ for 
arbitrary (constant) $\xi$, with gradient $\operatorname{grad}[\eta]=\widehat{\xi} D \varphi_{n+\alpha}$. In view of (3.3) and the symmetry of $S$ we conclude that

$$
\begin{aligned}
\left\langle\xi, \varphi_{n+\alpha} \times\left(\pi_{n+1}-\pi_{n}\right)\right\rangle= & \left\langle\xi \times \varphi_{n+\alpha}, \pi_{n+1}-\pi_{n}\right\rangle \\
= & \Delta t\left\langle\xi, \varphi_{n+\alpha} \times f_{n+\alpha}\right\rangle+\left\langle\xi, \varphi_{n+\alpha} \times \overline{\boldsymbol{t}}_{n+\alpha}\right\rangle_{\Gamma} \\
& -\Delta t\left\langle\operatorname{grad}\left[\boldsymbol{\xi} \times \boldsymbol{\varphi}_{n+\alpha}\right], D \boldsymbol{\varphi}_{n+\alpha} \boldsymbol{S}\right\rangle \\
= & \Delta t \boldsymbol{\xi} \cdot \boldsymbol{T}_{n+\alpha}^{\mathrm{ext}}-\Delta t\left\langle\hat{\xi}, D \boldsymbol{\varphi}_{n+\alpha} \boldsymbol{S} D \boldsymbol{\varphi}_{n+\alpha}^{T}\right\rangle \\
= & \Delta t \boldsymbol{\xi} \cdot \boldsymbol{T}_{n+\alpha}^{\mathrm{ext}} .
\end{aligned}
$$

The second term in (3.5) can be further simplified with the aid of (3.3b) to obtain

$$
\begin{aligned}
& \left\langle\xi,\left(\varphi_{n+1}-\varphi_{n}\right) \times\left(\alpha \pi_{n}+(1-\alpha) \pi_{n+1}\right)\right\rangle \\
& =\Delta t\left\langle\xi, V_{n+\alpha} \times\left(2 \pi_{n+(1 / 2)}-\pi_{n+\alpha}\right)\right\rangle \\
& =2 \Delta t\left\langle\xi, \varrho_{0} V_{n+\alpha} \times V_{n+(1 / 2)}\right\rangle .
\end{aligned}
$$

Inserting the last two expressions into (3.5) gives the result

$$
\boldsymbol{\xi} \cdot\left(\boldsymbol{J}_{n+1}-\boldsymbol{J}_{n}\right)=\Delta t \boldsymbol{\xi} \cdot \boldsymbol{T}_{n+\alpha}^{\mathrm{ext}}+2 \Delta t\left\langle\boldsymbol{\xi}, \varrho_{0} \boldsymbol{V}_{n+\alpha} \times \boldsymbol{V}_{n+(1 / 2)}\right\rangle,
$$

which collapses to $(3.4)_{2}$ if $\alpha=\frac{1}{2}$ and completes the proof.

Observe that exact conservation of the total linear momentum and the total angular momentum alone does not even guarantee consistency of the algorithm. As the preceding proof clearly shows, one can assign arbitrary values to the convected stress tensor $S$ without altering the linear and the angular momentum within the step.

\subsubsection{Algorithmic energy balance within a time step}

The following results are independent of the specific form of the constitutive equation for the convected stress tensor $\boldsymbol{S}_{t}$. Within a typical time interval $\left[t_{n}, t_{n+1}\right]$, the class of exact momentum-conserving algorithms obtained by setting $\alpha=\frac{1}{2}$ in (3.3a,b) satisfies the (finite) incremental relation

$$
\begin{aligned}
\underbrace{\left[K_{n+1}-K_{n}\right]}_{\Delta \text { Kinetic Energy }} & +\underbrace{\left\langle\boldsymbol{S}, \boldsymbol{E}\left(\boldsymbol{\varphi}_{n+1}\right)-\boldsymbol{E}\left(\boldsymbol{\varphi}_{n}\right)\right\rangle}_{\Delta \text { Internal Stress Work }} \\
& +\underbrace{\left[-\left\langle\left(\boldsymbol{f}_{n+(1 / 2)}, \boldsymbol{\varphi}_{n+1}-\boldsymbol{\varphi}_{n}\right\rangle-\left\langle\overline{\boldsymbol{t}}_{n+(1 / 2)}, \boldsymbol{\varphi}_{n+1}-\boldsymbol{\varphi}_{n}\right\rangle_{\Gamma}\right]\right.}_{\Delta \text { Potential Energy of Loading }}=0,
\end{aligned}
$$

which can be viewed as the algorithmic counterpart of the expended power theorem (2.8) in the interval $\left[t_{n}, t_{n+1}\right]$. By comparing the continuum relation 
(2.8) with (3.9) we concluded that the change in the potential energy of the loading predicted by the algorithm is exact for dead loads. Likewise, the predicted change in kinetic energy is also exact. On the other hand, the change internal energy predicted by the algorithm depends on the form of the algorithmic approximation adopted for the constitutive equation which, so far, remains unspecified. To prove (3.9) we make use of the identity

$$
K_{n+1}-K_{n}=\left\langle\pi_{n+1}-\pi_{n}, V_{n+(1 / 2)}\right\rangle,
$$

which is easily verified by a direct computation, and use (2.5b) to derive the relation

$$
\begin{aligned}
\operatorname{sym}\left[D \varphi_{n+(1 / 2)}^{T} \operatorname{grad}\left[\Delta t V_{n+(1 / 2)}\right]\right] & =\operatorname{sym}\left[D \varphi_{n+(1 / 2)}^{T}\left(D \varphi_{n+1}-D \varphi_{n}\right)\right] \\
& =\frac{1}{2}\left[C\left(\varphi_{n+1}\right)-C\left(\varphi_{n}\right)\right]=E\left(\varphi_{n+1}\right)-E\left(\varphi_{n}\right) .
\end{aligned}
$$

Setting $\boldsymbol{\eta}=\boldsymbol{V}_{n+(1 / 2)}$ in the algorithmic weak form (3.3) and making use of these two results yields (3.9).

Finally, let $\Delta \mathscr{D}_{\left[t_{n}, t_{n+1}\right]}^{\text {int }} \geq 0$ denote the algorithmic approximation to the time integral $\int_{t_{n}+1}^{t_{n}+1} \mathscr{D}_{t}^{\text {int }} d t$ of the internal dissipation function. The algorithmic approximation (3.3) is then said to be consistent with the reduced dissipation inequality if the following discrete counterpart of (2.9) holds:

$$
\begin{aligned}
E_{n+1}^{\mathrm{int}}-E_{n}^{\mathrm{int}}= & \left\langle\boldsymbol{S}, \boldsymbol{E}\left(\boldsymbol{\varphi}_{n+1}\right)-\boldsymbol{E}\left(\boldsymbol{\varphi}_{n}\right)\right\rangle-\Delta \mathscr{D}\left[\mathscr{D}_{\left[t_{n}, t_{n+1}\right]}^{\mathrm{int}},\right. \\
& \text { with } \Delta \mathscr{D}_{\left[t_{n}, l_{n+1}\right]}^{\mathrm{int}} \geq 0 .
\end{aligned}
$$

By analogy with the continuum problem, we view condition (3.12) as a restriction placed on the algorithmic approximation to the constitutive equation for $\boldsymbol{S}$. In particular, in the absence of external loading [i.e., assuming condition $\left(\mathrm{A} 1^{*}\right)$ ], if (3.12) holds then by combining (3.9) and (3.12) we obtain

$$
E_{n+1}^{\mathrm{tot}}-E_{n}^{\mathrm{tot}}:=\left[K_{n+1}+E_{n+1}^{\mathrm{int}}\right]-\left[K_{n}+E_{n}^{\mathrm{int}}\right]=-\Delta \mathscr{D}_{\left[t_{n}, t_{n+1}\right]}^{\mathrm{int}} \leq 0,
$$

which implies the discrete conserving condition (3.1). For elastodynamics, the case considered below, $\Delta \mathscr{D}_{\left[t_{n}, t_{n+1}\right]}^{\mathrm{int}}=0$ and (3.13) yields the condition of exact energy conservation. Consequently, (3.12) is the crucial condition to be verified in the algorithmic approximation to the constitutive equations.

\subsection{An exact energy-momentum scheme for (smooth) elastodynamics}

We show below that for elastodynamics the constraint (3.1) of exact energy conservation provides an explicit algorithmic constitutive equation for the convected stress $S$ within a typical time interval $\left[t_{n}, t_{n+1}\right]$ that completely defines the algorithmic approximation $(3.3 \mathrm{a}, \mathrm{b})$. In what follows we 
use the notation

$$
C(\varphi):=D \varphi^{T} D \varphi \quad \text { and } \quad E(\varphi):=\frac{1}{2}[C(\varphi)-1] \text { for } \varphi \in Q,
$$

to designate the right Cauchy-Green tensor and the Lagrangrian strain tensors associated with given configuration, respectively. To motivate the treatment of general constitutive models we consider first the simpler situation afforded by a quadratic potential.

\subsubsection{Elastodynamics: The Saint Venant-Kirchhoff model}

A key insight into the structure of the algorithmic constitutive relations is gained by considering first the case of a quadratic stored energy function of the form

$$
\hat{e}(\boldsymbol{C}(\varphi))=\frac{1}{2} \boldsymbol{E}(\varphi) \cdot \mathbf{C} \boldsymbol{E}(\varphi), \quad \text { where } \mathbf{C}:=\lambda \mathbf{1} \otimes \mathbf{1}+2 \mu \boldsymbol{I},
$$

which defines the so-called Saint Venant-Kirchhoff material. The wellknown limitations of this model include failure of the poly-convexity condition and incorrect behavior for extreme strains; see e.g., Ciarlet [1988]. For the purpose of the present discussion, however, the model has the key property of leading to the stress-strain relation $S_{t}=\mathbf{C E}\left(\varphi_{t}\right)$ linear in the Lagrangian strain. As a result, using the identity

$$
\begin{aligned}
& E\left(\varphi_{n+1}\right) \cdot \mathrm{CE}\left(\varphi_{n+1}\right)-E\left(\varphi_{n}\right) \cdot \mathrm{CE}\left(\varphi_{n}\right) \\
& \quad=\left[E\left(\varphi_{n+1}\right)-E\left(\varphi_{n}\right)\right] \cdot \mathrm{C}\left[E\left(\varphi_{n+1}\right)+E\left(\varphi_{n}\right)\right],
\end{aligned}
$$

the change in internal energy within a time step $\left[t_{n}, t_{n+1}\right]$ is expressed as

$$
\begin{aligned}
E_{n+1}^{\mathrm{int}}-E_{n}^{\mathrm{int}} & :=\int_{\mathscr{B}}\left[\hat{e}\left(\boldsymbol{C}\left(\boldsymbol{\varphi}_{n+1}\right)\right)-\hat{e}\left(\boldsymbol{C}\left(\boldsymbol{\varphi}_{n}\right)\right) d \mathscr{B}\right. \\
& =\frac{1}{2}\left\langle\left[\boldsymbol{E}\left(\boldsymbol{\varphi}_{n+1}\right)-\boldsymbol{E}\left(\boldsymbol{\varphi}_{n}\right)\right], \boldsymbol{C}\left[\boldsymbol{E}\left(\boldsymbol{\varphi}_{n+1}\right)+\boldsymbol{E}\left(\boldsymbol{\varphi}_{n}\right)\right]\right\rangle .
\end{aligned}
$$

In view of (3.12) from (3.17) we conclude that for a stored energy function quadratic in the (nonlinear) Lagrangian strains exact energy conservation for dead loading is achieved if

$$
E_{n+1}^{\mathrm{int}}-E_{n}^{\mathrm{int}}=\left\langle\boldsymbol{S}, \boldsymbol{E}\left(\varphi_{n+1}\right)-\boldsymbol{E}\left(\varphi_{n}\right)\right\rangle \Leftrightarrow S=\mathbf{C}\left[\boldsymbol{E}\left(\varphi_{n+1}\right)+E\left(\varphi_{n}\right)\right] / 2 .
$$

This result is unexpected. Since the internal energy term is evaluated at the mid-point configuration $\varphi_{n+(1 / 2)} \in Q$ it would be natural to adopt the constitutive equation $\boldsymbol{S}=\mathbf{C} \boldsymbol{E}\left(\boldsymbol{\varphi}_{n+(1 / 2)}\right)$. However, in sharp constrast with the result obtained in (3.18), this choice would result in failure of the property exact energy conservation in an algorithmic setting. In other words, for the Saint Venant-Kirchhoff model, use of the average of the 
strains and not the strain of the average configuration is the key to exact enforcement exact energy conservation.

\subsubsection{Elastodynamics: General constitutive models}

The preceding result is extended to general elastic constitutive equations as follows. Define the algorithmic right Cauchy-Green tensor and the Lagrangian strain tensor at the configuration $\varphi_{n+\beta} \in Q$ via the convex combination

$$
C_{n+\beta}:=\beta C\left(\varphi_{n+1}\right)+(1-\beta) C\left(\varphi_{n}\right), \beta \in[0,1] \text { and } E_{n+\beta}:=\frac{1}{2}\left[C_{n+\beta}-1\right]
$$

Observe carefully that, in general, $\boldsymbol{C}_{n+\beta} \neq \boldsymbol{C}\left(\boldsymbol{\varphi}_{n+\beta}\right)$ and $\boldsymbol{E}_{n+\beta} \neq \boldsymbol{E}\left(\boldsymbol{\varphi}_{n+\beta}\right)$ except for $\beta=0$ and $\beta=1$. Nevertheless the convex approximation (3.19) retains the two crucial properties of the right Cauchy-Green tensor; namely, symmetry and positive definiteness, since

$$
C_{n+\beta}=C_{n+\beta}^{T} \quad \text { and } \quad \xi \cdot C_{n+\beta} \xi>0 \quad \xi \in \mathbb{R}^{n_{\mathrm{dim}}},
$$

for all $\beta \in[0,1]$.

The key result exploited in the enforcement of energy conservation is a direct consequence of the mean value theorem (see e.g. Marsden [1974]). The convex combination (3.19) has the property that $(d / d \beta) C_{n+\beta}=$ $C\left(\varphi_{n+1}\right)-C\left(\varphi_{n}\right)$. Consequently, by the mean value theorem, there exist $\beta_{0} \in[0,1]$ such that

$$
\begin{aligned}
\hat{e}\left(\boldsymbol{C}_{n+1}\right)-\hat{e}\left(\boldsymbol{C}_{n}\right) & =\left.\frac{d}{d \beta}\right|_{\beta=\beta_{0}} \hat{e}\left(\boldsymbol{C}_{n+\beta}\right) \\
& =\left.\nabla \hat{e}\left(\boldsymbol{C}_{n+\beta_{0}}\right) \cdot \frac{d \boldsymbol{C}_{n+\beta}}{d \beta}\right|_{\beta=\beta_{0}} \\
& =\nabla \hat{e}\left(\boldsymbol{C}_{n+\beta_{0}}\right) \cdot\left[\left(\boldsymbol{C}_{n+1}-\boldsymbol{C}_{n}\right)\right] .
\end{aligned}
$$

Now, under the assumption of dead loading, exact conservation of energy for a general stored function $\hat{e}\left(C\left(\varphi_{t}\right)\right)$ holds if condition (3.12) holds with $\Delta \mathscr{D}_{\left[t_{n}, t_{n+1}\right]}^{\mathrm{int}} \equiv 0$; i.e.,

$$
\int_{\mathscr{B}}\left[\hat{e}\left(\boldsymbol{C}\left(\boldsymbol{\varphi}_{n+1}\right)\right)-\hat{e}\left(\boldsymbol{C}\left(\boldsymbol{\varphi}_{n}\right)\right)\right] d \mathscr{B}=\frac{1}{2}\left\langle\boldsymbol{S}, \boldsymbol{C}\left(\boldsymbol{\varphi}_{n+1}\right)-\boldsymbol{C}\left(\boldsymbol{\varphi}_{n}\right)\right\rangle .
$$

In view of (3.21) and (3.22) we conclude that energy conservation is exactly enforced, without upsetting conservation of total linear and total angular 
momentum, by adopting the algorithmic constitutive equation

$$
\begin{aligned}
& \boldsymbol{S}=2 \nabla \hat{e}\left(\boldsymbol{C}_{n+\beta_{0}}\right) \quad \text { with } \quad \beta_{0} \in(0,1) \text { such that: } \\
& \hat{e}\left(\boldsymbol{C}_{n+1}\right)-\hat{e}\left(\boldsymbol{C}_{n}\right)=\boldsymbol{S} \cdot \frac{1}{2}\left(\boldsymbol{C}_{n+1}-\boldsymbol{C}_{n}\right)
\end{aligned}
$$

For general constitutive models it is shown below that the actual determination of $\beta_{0}$ can be performed essentially at no additional cost as part of the standard iterative solution process for the (finite element form) of the momentum equation.

\section{Remarks 3.1.}

1. The existence of the required $\beta_{0} \in(0,1)$ in $(3.23)$ is guaranteed by the mean value theorem. In particular, if $\hat{e}(\cdot)$ is quadratic we have $\beta_{0}=\frac{1}{2}$ is agreement with our analysis for the Saint Venant-Kirchhoff model.

2. Second order accurate algorithms. An accuracy analysis identical to that presented in Appendix I shows that the algorithm defined by $(3.3 \mathrm{a}, \mathrm{b})$ along with (3.23) is second order accurate if and only if $\alpha=\beta_{0}=\frac{1}{2}$. Thus, if the algorithmic constitutive equation (3.23) is adopted, exact energy conservation and second order accuracy can only be achieved for the Saint Venant-Kirchhoff model. This limitation is removed as follows. Define the function $g:[0,1] \rightarrow \mathbb{R}$ by setting (see Figure 3.2)

$$
g(\beta):=\frac{1}{2}\left[\hat{e}\left(\boldsymbol{C}_{n+\beta}\right)-\hat{e}\left(\boldsymbol{C}_{n+(1-\beta)}\right)\right] .
$$

Figure 3.2

The mean value theorem applied to the odd function $g(\cdot)$ defined in Remark 3.1.2. Here $\beta_{0} \in\left(0, \frac{1}{2}\right]$ always exists such that $g(1)-g(0)=$ $g^{\prime}\left(\beta_{0}\right)$.

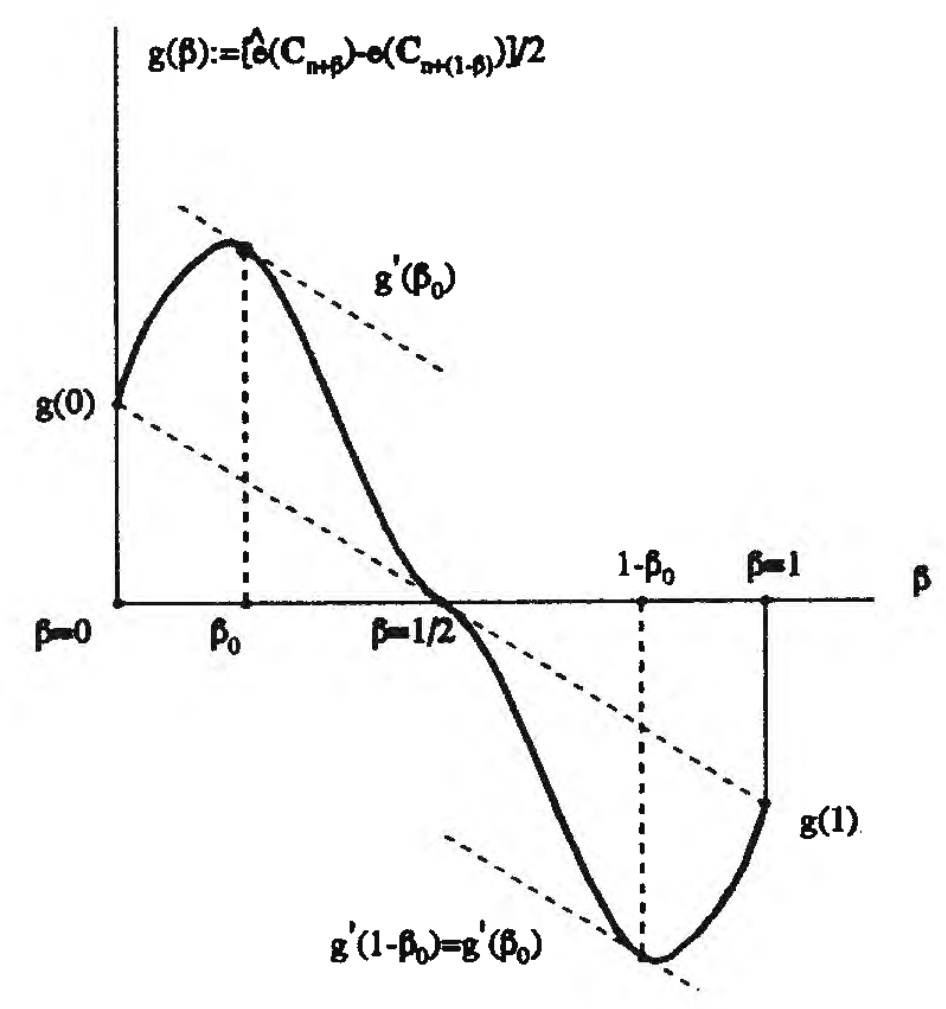


Clearly, $g(\cdot)$ is odd about $\beta=\frac{1}{2}$; i.e., it satisfies the property

$$
g(\beta)=-g(1-\beta) \quad \forall \beta \in[0,1] \Rightarrow g(1)-g(0)=\hat{e}\left(C_{n+1}\right)-\hat{e}\left(C_{n}\right) .
$$

By the mean value theorem, there exists $\beta_{0} \in(0,1)$ such that $g(1)-g(0)=$ $g^{\prime}\left(\beta_{0}\right)$. Property $(3.25)_{1}$ then implies that the mean value theorem is also satisfied for $1-\beta_{0} \in(0,1)$. Setting

$$
S:=\nabla \hat{e}\left(C_{n+\beta_{0}}\right)+\nabla \hat{e}\left(C_{n+\left(1-\beta_{0}\right)}\right),
$$

from $(3.25)_{1}$ and relation $g(1)-g(0)=g^{\prime}\left(\beta_{0}\right)=g^{\prime}\left(1-\beta_{0}\right)$ we conclude that $\hat{e}\left(\boldsymbol{C}_{n+1}\right)-\hat{e}\left(\boldsymbol{C}_{n}\right)=\boldsymbol{S} \cdot \frac{1}{2}\left[\boldsymbol{C}_{n+1}-\boldsymbol{C}_{n}\right]$. Therefore, the algorithmic constitutive equation also yields exact energy conservation [for a suitable $\beta_{0} \in(0,1)$ ] but, in contrast with relation (3.23), the resulting exact energy-momentum conserving algorithm retains the second order accuracy property even if $\beta_{0} \neq \frac{1}{2}$. See Appendix I for a detailed accuracy analysis.

3. The energy-momentum conserving algorithm described above is clearly consistent. Unconditional (formal) nonlinear stability follows from exact energy conservation since, formally, the total energy defines a Lyapunov function for the dynamics.

\section{§4. Spatial discretization: Galerkin FEM projection}

We examine below the Galerkin projection of the algorithmic weak form $(3.3 \mathrm{a}, \mathrm{b})$ of the temporal discretization of the IBVP onto a finite dimensional phase and show that this projection preserves the momentum maps. Moreover, we show that the time discretization of the weak form commutes with the spatial Galerkin projection. Consequently, all the conservation properties derived in the preceding Section are inherited by the discrete (algebraic) finite element problem. For completeness, key aspects involved in the actual finite element implementation of the exact-momentum conserving algorithm for elastodynamics are briefly summarized.

\subsection{The Galerkin FEM spatial projection}

Let $\mathscr{S}$ denote the (infinite dimensional) state space for the problem at hand, consisting of admissible configurations and material velocity fields on $\mathscr{B}$, defined as

$$
\mathscr{S}:=\left\{(\boldsymbol{\varphi}, \boldsymbol{V}): \mathscr{B} \rightarrow \mathbb{R}^{n_{\mathrm{dim}}} \times \mathbb{R}^{n_{\mathrm{dim}}} \mid \boldsymbol{\varphi} \in Q \text { and }\left.\boldsymbol{V}\right|_{\Gamma_{\sigma}}=\mathbf{0}\right\} .
$$

We denote by $N^{A}: \mathscr{B} \rightarrow \mathbb{R}$ the prescribed $n_{\text {node }}$ global finite element shape functions of a finite element discretization of the reference placement $\mathscr{B}$, 
with nodal points $\boldsymbol{X}_{A} \in \overline{\mathscr{B}}, A=1, \ldots, n_{\text {node }}$. As usual we assume the (completeness) condition $N^{A}\left(\boldsymbol{X}_{B}\right)=\delta_{B}^{A}$. The Galerkin projection of the state space $\mathscr{S}$ onto a finite dimensional state subspace space $\mathscr{S}^{h} \subset \mathscr{S}$ is defined via the formulae

$$
\boldsymbol{\varphi}^{h}(\boldsymbol{X})=\sum_{A=1}^{n_{\text {node }}} N^{A}(\boldsymbol{X}) \boldsymbol{d}_{A} \quad \text { and } \quad \boldsymbol{V}^{h}(\boldsymbol{X})=\sum_{A=1}^{n_{\text {node }}} N^{A}(\boldsymbol{X}) \boldsymbol{V}_{A}, \quad \forall \boldsymbol{X} \in \overline{\mathscr{B}},
$$

where $\left(\varphi^{h}, V^{h}\right) \in \mathscr{S}^{h}$ and $(\varphi, V) \in \mathscr{S}$ is an arbitrary point in the state space. The dimension of $\mathscr{S}^{h}$ is $n_{\text {ndof }} \times n_{\text {ndof }}$ where $n_{\text {ndof }}:=n_{\text {dim }} \times n_{\text {node }}$. Similarly, the finite element subspace $\mathscr{V}^{h} \subset \mathscr{V}$ of admissible test functions is defined by

$$
\mathscr{V}^{h}:=\left\{\boldsymbol{\eta}^{h} \in \mathscr{V} \mid \boldsymbol{\eta}^{h}=\sum_{A=1}^{n_{\text {node }}} N^{A}(\boldsymbol{X}) \boldsymbol{c}_{A}, \boldsymbol{c}_{A} \in \mathbb{R}^{n_{\mathrm{dim}}} \text { for } A=1, \ldots, n_{\mathrm{dim}}\right\} .
$$

In what follows we shall use the notation $M^{A B}:=\left\langle N^{A}, \varrho_{0} N^{B}\right\rangle=M^{B A}$ to designate the entries of the "mass matrix" of the finite element discretization.

\subsubsection{Projected algorithmic dynamics and momentum maps}

By inserting the spatial interpolations defined by (4.2) and (4.3) into the time-discretization (3.3a) of the weak form of balance of momentum we obtain a nonlinear algebraic equation defined by the expression

$$
\left.\begin{array}{r}
\sum_{B=1}^{n_{\text {node }}} M^{A B} \frac{1}{\Delta t}\left[\boldsymbol{V}_{n+1 B}-\boldsymbol{V}_{n B}\right]+\boldsymbol{F}_{n+\alpha}^{\mathrm{int} A}=\boldsymbol{F}_{n+\alpha}^{\mathrm{ext} A}, \\
\frac{1}{\Delta t}\left[\boldsymbol{d}_{n+1 A}-\boldsymbol{d}_{n A}\right]=\boldsymbol{V}_{n+\alpha A},
\end{array}\right\}
$$

for $A=1,2, \ldots, n_{\text {node }}$, where $\boldsymbol{F}_{n+\alpha}^{\mathrm{int} A}$ and $\boldsymbol{F}_{n+\alpha}^{\mathrm{ext} A}$ denote the internal and external forces associated with node $X_{A}$ at time $t_{n+\alpha}$, as defined by the relations

$$
\left.\begin{array}{rl}
\boldsymbol{F}_{n+\alpha}^{\mathrm{int} A} & :=\int_{\mathscr{B}} D \boldsymbol{\varphi}_{n+\alpha}^{h} \boldsymbol{S}^{h} \operatorname{grad}\left[N^{A}\right] d \mathscr{B}, \\
\boldsymbol{F}_{n+\alpha}^{\mathrm{ext} A} & :=\int_{\mathscr{B}} N^{A} \boldsymbol{f}_{n+\alpha} d \mathscr{B}+\int_{\Gamma_{\sigma}} N^{A} \overline{\boldsymbol{t}}_{n+\alpha} d \Gamma .
\end{array}\right\}
$$

Consider next the momentum conservation properties inherited by the algebraic problem (4.4). By inserting the interpolations defined by (4.2) into definitions (2.15) gives the following expressions for the projected total 
linear momentum and the projected total angular momentum:

$$
\boldsymbol{L}_{t}^{h}:=\sum_{A=1}^{n_{\text {node }}}\left\langle\varrho_{0}, N^{A}\right\rangle \boldsymbol{V}_{t A} \quad \text { and } \quad \boldsymbol{J}_{t}^{h}:=\sum_{A=1}^{n_{\text {node }}} \sum_{B=1}^{n_{\text {node }}} M^{A B} \boldsymbol{d}_{t A} \times \boldsymbol{V}_{t B}, \quad \forall t \in \mathbb{\mathbb { V }} .
$$

Now let $\boldsymbol{L}_{n}^{h}, \boldsymbol{L}_{n+1}^{h}$ and $\boldsymbol{J}_{n}^{h}, \boldsymbol{J}_{n+1}^{h}$ denote the total linear and total angular momentum at times $t_{n}$ and $t_{n+1}$, respectively, obtained by evaluating expressions (4.6) for the nodal values $\left(\boldsymbol{d}_{n}, \boldsymbol{V}_{n}\right)$ and $\left(\boldsymbol{d}_{n+1}, \boldsymbol{V}_{n+1}\right)$. If $\left(\boldsymbol{d}_{n+1}, \boldsymbol{V}_{n+1}\right) \in \mathbb{R}^{n_{\text {node }}} \times \mathbb{R}^{n_{\text {node }}}$ satisfies the algebraic problem (4.4) then for the Neumann problem [i.e., under assumption (A2)] the following relations holds

$$
\left.\begin{array}{ll}
\boldsymbol{L}_{n+1}^{h}-\boldsymbol{L}_{n}^{h}=\Delta t \sum_{A=1}^{n_{\text {node }}} \boldsymbol{F}_{n+\alpha}^{\mathrm{ext} A} & \text { for any } \alpha \in[0,1], \\
\boldsymbol{J}_{n+1}^{h}-\boldsymbol{J}_{n}^{h}=\Delta t \sum_{A=1}^{n_{\text {node }}} \boldsymbol{d}_{n+\alpha A} \times \boldsymbol{F}_{n+\alpha}^{\mathrm{ext} A} & \text { only if } \alpha=\frac{1}{2} .
\end{array}\right\}
$$

In particular, in the absence of external loads [i.e., under assumption (A1*)] for the value $\alpha=\frac{1}{2}$ one recovers the discrete conservation laws of linear and angular momentum; namely, $\boldsymbol{L}_{n+1}^{h}=\boldsymbol{L}_{n}^{h}$ and $\boldsymbol{J}_{n+1}^{h}=\boldsymbol{J}_{n}^{h}$. The proof of the discrete relations (4.7) relies crucially on the following invariance condition

$$
\sum_{A=1}^{n_{\text {node }}} \boldsymbol{d}_{n+\alpha A} \times \boldsymbol{F}_{n+\alpha}^{\mathrm{int} A}=\mathbf{0} \text { for any } \alpha \in[0,1],
$$

which follows from the symmetry of $\boldsymbol{S}^{h}$ since

$$
\begin{aligned}
\xi \cdot \sum_{A=1}^{n_{\text {node }}} d_{n+\alpha A} \times \boldsymbol{F}_{t}^{\text {int } A} & =\left\langle\sum_{A=1}^{n_{\text {node }}}\left[\left(\boldsymbol{\xi} \times \boldsymbol{d}_{n+\alpha A}\right) \otimes \operatorname{grad}\left[N^{A}\right]\right], D \boldsymbol{\varphi}_{n+\alpha}^{h} \boldsymbol{S}^{h}\right\rangle \\
& =\left\langle\hat{\xi}, D \boldsymbol{\varphi}_{n+\alpha}^{h} \boldsymbol{S}^{h} D \boldsymbol{\varphi}_{n+\alpha}^{h T}\right\rangle=0,
\end{aligned}
$$

for any vector $\xi \in \mathbb{R}^{3}$. With this result in hand, the proof of (4.7) follows along the same lines as the argument presented in Section 3.2 for the time-discrete continuous problem and further details are omitted. Recall that $\boldsymbol{S}^{h}=\boldsymbol{S}^{h T}$ remains so far arbitrary.

It can be easily verified that a result identical to that in (4.4) is obtained if the Galerkin projection is applied first to the continuous weak form $(2.5 \mathrm{a}, \mathrm{b})$ of the momentum equation, followed by the time discretization. Therefore, the following diagram commutes:

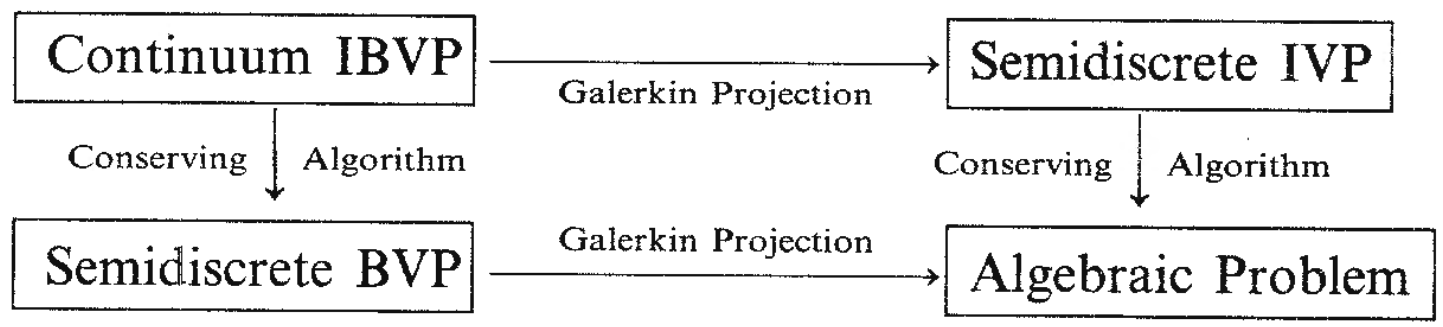


Furthermore, this diagram preserves the momentum maps in the sense that the spatial and temporal discretizations of the conservation laws of momentum, applied in any order, define the conservation laws of the discrete algebraic problem (for $\alpha=\frac{1}{2}$ ).

\subsubsection{Algorithmic dissipation and energy balance within a time step}

It can be easily shown that the incremental form (3.9) of the expended power theorem also holds in $\mathscr{S}^{h}$. Again the result is independent of the order in which the Galerkin interpolation and the (conserving) time discretization are applied. Similarly, the algorithmic approximation restricted to $\mathscr{S}^{h}$ is said to be consistent with the reduced dissipation inequality if the counterpart of (3.12) holds; i.e.,

$$
\Delta \mathscr{D}_{\left[t_{n}, t_{n+1}\right]}^{\mathrm{int}}:=\left\langle\boldsymbol{S}^{h}, \boldsymbol{E}\left(\varphi_{n+1}^{h}\right)-\boldsymbol{E}\left(\varphi_{n}^{h}\right)\right\rangle-\left[E_{n+1}^{\mathrm{int} h}-E_{n}^{\mathrm{int} h}\right] \geq 0 .
$$

As in the time semi-discrete case, in the absence of external loading [i.e., assumption $\left.\left(\mathrm{A} 1^{*}\right)\right]$ the total energy $E^{\text {tot } h}:=K^{h}+E^{\text {int } h}$ computed with an algorithm for which condition (4.10) holds satisfies the a-priori estimate

$$
E_{n+1}^{\text {tot } h}-E_{n}^{\text {tot } h}:=-\Delta \mathscr{D}_{\left[n, t_{n+1}\right]}^{\text {int } h} \leq 0 \quad \text { in } \mathscr{S}^{h} \times\left[t_{n}, t_{n+1}\right] .
$$

For elastodynamics the total energy is conserved if (4.10) holds with $\Delta \mathscr{D}_{\left[t_{n}, t_{n+1}\right]}^{\text {int } h} \equiv 0$. This is the case for the algorithmic constitutive equations (3.23) now evaluated on $Q^{h}$.

In summary, for elastodynamics the Galerkin finite element projection of the dynamics onto a finite dimensional phase space $\mathscr{S}^{h}$ preserves all the algorithmic conservation properties derived in the preceding section and commutes with the time discretization. The situation is not nearly as transparent in other models of continuum physics, such as nonlinear rods and shells, where straightforward Galerkin projection may not preserve the momentum maps and spoil, therefore, algorithmic conservation properties. We refer to Simo, Rifai and Fox [1992] for a detailed analysis of these and related issues in the context of nonlinear shells.

\subsection{Implementation. Remarks on computational aspects}

We outline below the steps within a typical time subinterval $\left[t_{n}, t_{n+1}\right]$ involved in the actual implementation of the exact energy-momentum conserving algorithm for nonlinear elastodynamics. Remarkably, this implementation is essentially identical to that of standard algorithms; The only additional effort required being the local solution (at quadrature points) of a scalar nonlinear equation. Clearly this addition involves negligible cost. 
Assume that $\left(\boldsymbol{d}_{n A}, V_{n A}\right)$ are prescribed initial data at the nodal points and time $t_{n}$, for $A=1,2, \ldots, n_{\text {node }}$. Since the discrete problem (4.4) along with constitutive equations (3.23) is nonlinear, an iterative solution strategy must be adopted. In what follows the approximate solution of (4.4) at the $k$ th iteration within the time will be denoted simply by $\left(d_{n+1 A}, V_{n+1 A}\right)$.

Step 1. Given $\left(\boldsymbol{d}_{n+1 A}, \boldsymbol{V}_{n+1 A}\right),\left(A=1,2, \ldots, n_{\text {node }}\right)$, compute the projected deformation gradients $D \varphi_{n}^{h}, D \varphi_{n+(1 / 2)}^{h}$ and $D \varphi_{n+1}^{h}$ by evaluation of the general formula

$D \varphi_{n+\alpha}^{h}=\sum_{A=1}^{n_{\text {noce }}}\left[\alpha d_{n+1 A}+(1-\alpha) d_{n A}\right] \otimes \operatorname{grad}\left[N^{A}\right]$

for $\alpha=0, \alpha=\frac{1}{2}$ and $\alpha=1$, respectively. Then compute the right Cauchy-Green tensors: $C_{n}^{h}=D \varphi_{n}^{h T} D \varphi_{n}^{h}$ and $C_{n+1}^{h}=$ $D \varphi_{n+1}^{h T} D \varphi_{n+1}^{h}$.

Step 2. For fixed $\boldsymbol{C}_{n+1}^{h}$ (defined above) and given $\boldsymbol{C}_{n}^{h}$ define the (generally) nonlinear function $g^{\prime}:(0,1) \rightarrow \mathbb{R}$ as

$$
\begin{aligned}
g^{\prime}(\beta):= & \hat{e}\left(\boldsymbol{C}_{n+1}^{h}\right)-\hat{e}\left(\boldsymbol{C}_{n}^{h}\right) \\
& -\frac{1}{2}\left[\nabla \hat{e}\left(\boldsymbol{C}_{n+\beta}^{h}\right)+\nabla \hat{e}\left(\boldsymbol{C}_{n+(1-\beta)}^{h}\right)\right] \cdot\left(\boldsymbol{C}_{n+1}^{h}-\boldsymbol{C}_{n}^{h}\right),
\end{aligned}
$$

where $C_{n+\alpha}^{h}:=\alpha C_{n+1}^{h}+(1-\alpha) C_{n}^{h}$ is evaluated at $\alpha=\beta$ and $\alpha=1-\beta$ in $(0,1)$, respectively. Then find the value $\beta_{0} \in(0,1)$ closest to $\frac{1}{2}$ such that $g^{\prime}\left(\beta_{0}\right)=0$. For the Saint Venant-Kirchhoff model $\beta_{0}=\frac{1}{2}$.

Step 3. Evaluate the convected stress tensor and the convected moduli by the algorithmic formulae

$$
\left.\begin{array}{l}
\boldsymbol{S}^{h}:=\nabla \hat{e}\left(\boldsymbol{C}_{n+\beta_{0}}^{h}\right)+\nabla \hat{e}\left(\boldsymbol{C}_{n+\left(1-\beta_{0}\right)}^{h}\right), \\
\mathbf{C}^{h}:=2\left[\beta_{0} \nabla^{2}\left[\hat{e}\left(\boldsymbol{C}_{n+\beta_{0}}^{h}\right)+\left(1-\beta_{0}\right) \nabla^{2} \hat{e}\left(\boldsymbol{C}_{n+\left(1-\beta_{0}\right)}^{h}\right)\right] .\right.
\end{array}\right\}
$$

Then compute $\boldsymbol{F}_{n+(1 / 2)}^{\text {int }}$ and $\boldsymbol{F}_{n+(1 / 2)}^{\text {ext }}$ via formulae (4.5) and evaluate the dynamic residual as

$\boldsymbol{R}^{A}:=\sum_{B=1}^{n_{\text {node }}} M^{A B} \frac{2}{\Delta t^{2}}\left[\boldsymbol{d}_{n+1 B}-\boldsymbol{d}_{n B}-\Delta t \boldsymbol{V}_{n B}\right]+\left[\boldsymbol{F}_{n+(1 / 2)}^{\mathrm{int} A}-\boldsymbol{F}_{n+(1 / 2)}^{\mathrm{ext} A}\right]$.

If $\left\|\boldsymbol{R}^{A}\right\| \leq T O L$ then $\left(\boldsymbol{d}_{n+1 A}, \boldsymbol{V}_{n+1 A}\right)$ is the sought solution.

Step 4. If $\left\|\boldsymbol{R}^{A}\right\|>T O L$ then solve for $\Delta \boldsymbol{d}_{n+1 A}$ the linearized system

$\sum_{B=1}^{n_{\text {node }}}\left[\frac{2}{\Delta t^{2}} M^{A B} \mathbf{1}+\boldsymbol{K}^{A B}\right] \Delta \boldsymbol{d}_{n+1 B}+\boldsymbol{R}^{A}=\mathbf{0}$.

Update the solution as $\boldsymbol{d}_{n+1 A} \leftarrow \boldsymbol{d}_{n+1 A}+\Delta \boldsymbol{d}_{n+1 A}$ and return to Step 1. 


\section{Remarks 4.1}

1. The scalar equation $g^{\prime}(\beta)=0$ in Step 2 is easily solved since the solution $\beta_{0} \in(0,1)$ is precisely bounded. Thus any variant of Newton's method could be used, in conjunction with line search due to the lack of convexity of the stored energy $\hat{e}(\cdot)$. Observe that the derivative of $g^{\prime \prime}(\beta)$ is trivially computed by the closed-form formula

$$
g^{\prime \prime}(\beta)=-\frac{1}{2}\left(\boldsymbol{C}_{n+1}^{h}-\boldsymbol{C}_{n}^{h}\right) \cdot\left[\nabla^{2} \hat{e}\left(\boldsymbol{C}_{n+\beta}^{h}\right)-\nabla^{2} \hat{e}\left(\boldsymbol{C}_{n+(1-\beta)}^{h}\right)\right]\left(\boldsymbol{C}_{n+1}^{h}-\boldsymbol{C}_{n}^{h}\right) .
$$

2. Strictly speaking, the matrix $\boldsymbol{K}^{A B}$ obtained by exact linearization of the internal force vector $\boldsymbol{F}_{n+(1 / 2)}^{\text {int } A}$ is non-symmetric. In fact a standard calculation readily gives the expression

$$
\begin{aligned}
\boldsymbol{K}^{(k) A B}= & \int_{\mathscr{B}} \underbrace{\left[\left(\operatorname{grad}\left[N^{A}\right] \cdot \boldsymbol{S}^{h} \operatorname{grad}\left[N^{B}\right]\right) 1\right.}_{\text {Geometric Term }} \\
& +\underbrace{\left(\left[D \varphi_{n+(1 / 2)}^{h T} \operatorname{grad}\left[N^{A}\right]\right) \mathbf{C}^{h}\left(D \boldsymbol{\varphi}_{n+\beta_{0}}^{h T} \operatorname{grad}\left[N^{B}\right]\right)\right] d \mathscr{B}}_{\text {Material Term }},
\end{aligned}
$$

which shows that the lack of symmetry is associated with the material term arising for the linearization of the algorithmic constitutive equation (4.14) ${ }_{1}$. We observe that this lack of symmetry is 'mild' and disappears as $\Delta t \rightarrow 0$.

\section{§5. Representative numerical simulations}

The goal of this Section is to assess the performance exhibited by energy-momentum algorithm described above relative to two representative algorithms: The widely used trapezoidal rule and the symplectic midpointrule, shown in Simo, Tarnow and Wong [1992] to define an exact momentum preserving scheme. The trapezoidal rule (note the mid-point rule) can be obtained by setting $\beta_{0}=\frac{1}{4}$ and $\gamma=\frac{1}{2}$ the classical Newmark family. We consider two representative simulations that exhibit finite deformations coupled with large overall rigid body motion.

\subsection{Dynamics of a tumbling L-shaped block}

In this first example we consider the dynamics of an L-shaped block subject to no boundary restrictions with material response governed by a Saint Venant-Kirchhoff elastic model. After an initial loading phase the 
block tumbles free of external forces in the ambient space undergoing finite deformation accompanied by large overall rotations and translations. All the data required to define the problem and perform the simulation are summarized in Figure 5.1.

According to our analysis in Section 2 the algorithmic constitutive equation $\boldsymbol{S}^{h}=\mathbf{C} \frac{1}{2}\left[\boldsymbol{E}\left(\varphi_{n+1}^{h}\right)+\boldsymbol{E}\left(\varphi_{n}^{h}\right)\right]$ for the second Piola-Kirchhoff tensor along with the algorithmic weak form (3.3a,b) particularized at $\alpha=\frac{1}{2}$ defines an exact energy-momentum conserving scheme. A main goal of this simulation is to verify numerically these properties. The sequence of deformed shapes obtained in the course of the simulation, shown in Figure 5.2 without any magnification of the actual deformations, clearly demonstrates the presence of finite deformations accompanied by a large overall rigid motion.

The sequence of plots summarized in Figure 5.3 contain the time histories total energy and the three components of the total angular momentum computed with the energy-momentum algorithm, the symplectic midpoint-rule and the trapezoidal rule of the Newmark family, respectively. The time history of the total linear momentum is not reported since these

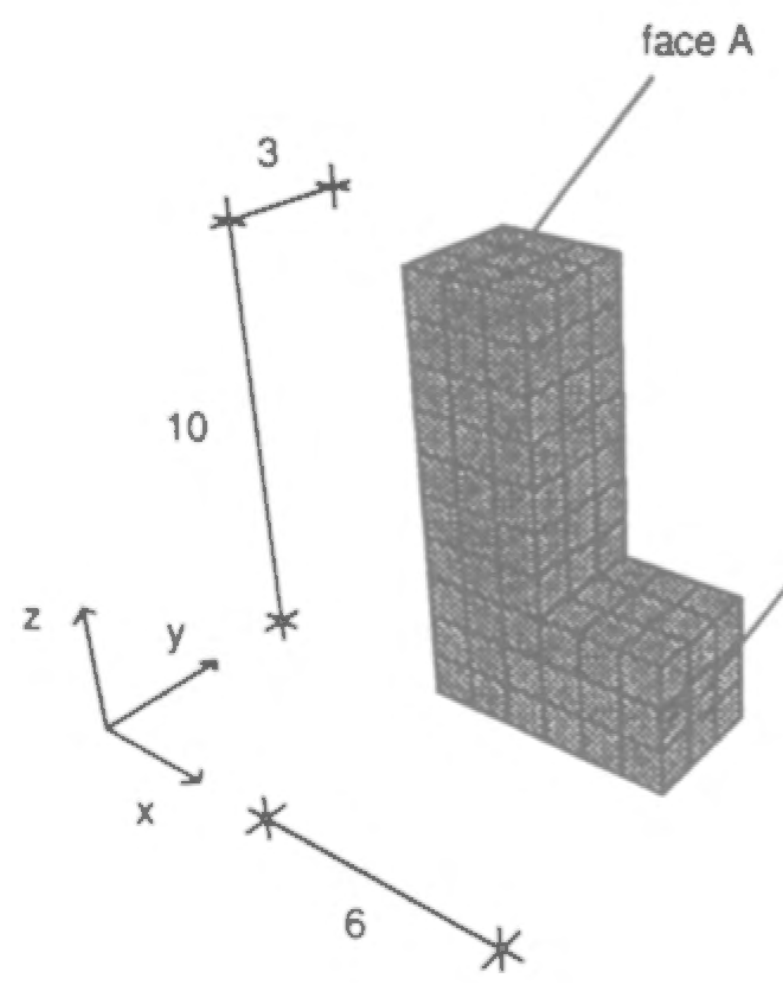

$$
\text { resultant loads } \quad \text { on face A }=\left\{\begin{array}{l}
F_{x}=1600 \cdot p(t) \\
F_{y}=800 \cdot \cdot p(t) \\
F_{z}=1600 \cdot p(t) \\
M_{x}=0 . \\
M_{y}=0 . \\
M_{z}=-1200 \cdot \cdot p(t)
\end{array}\right.
$$

face B

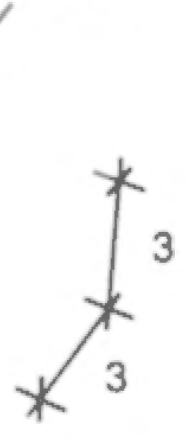

resultant loads $\quad$ on faceB $=\left\{\begin{array}{l}F_{x}=800 \cdot p(t) \\ F_{y}=-1600 \cdot p(t) \\ F_{z}=-800 \cdot p(t) \\ M_{x}=-1200 \cdot p(t) \\ M_{y}=0 . \\ M_{z}=0 .\end{array}\right.$

$p(t)=\left\{\begin{array}{rll}t & \text { for } & t \leq 0.5 \\ 0.5-t & \text { for } & 0.5 \leq t \leq 1.0 \\ 0 . & \text { for } & t \geq 1.0\end{array}\right.$

Figure 5.1

Geometry and finite element mesh for the simulation of the dynamics of a 'tumbling' L-shape elastic block with material response governed by the Saint Venant-Kirchhoff elastic model. The body is initially at rest and then subjected to the loading given in the figure. 


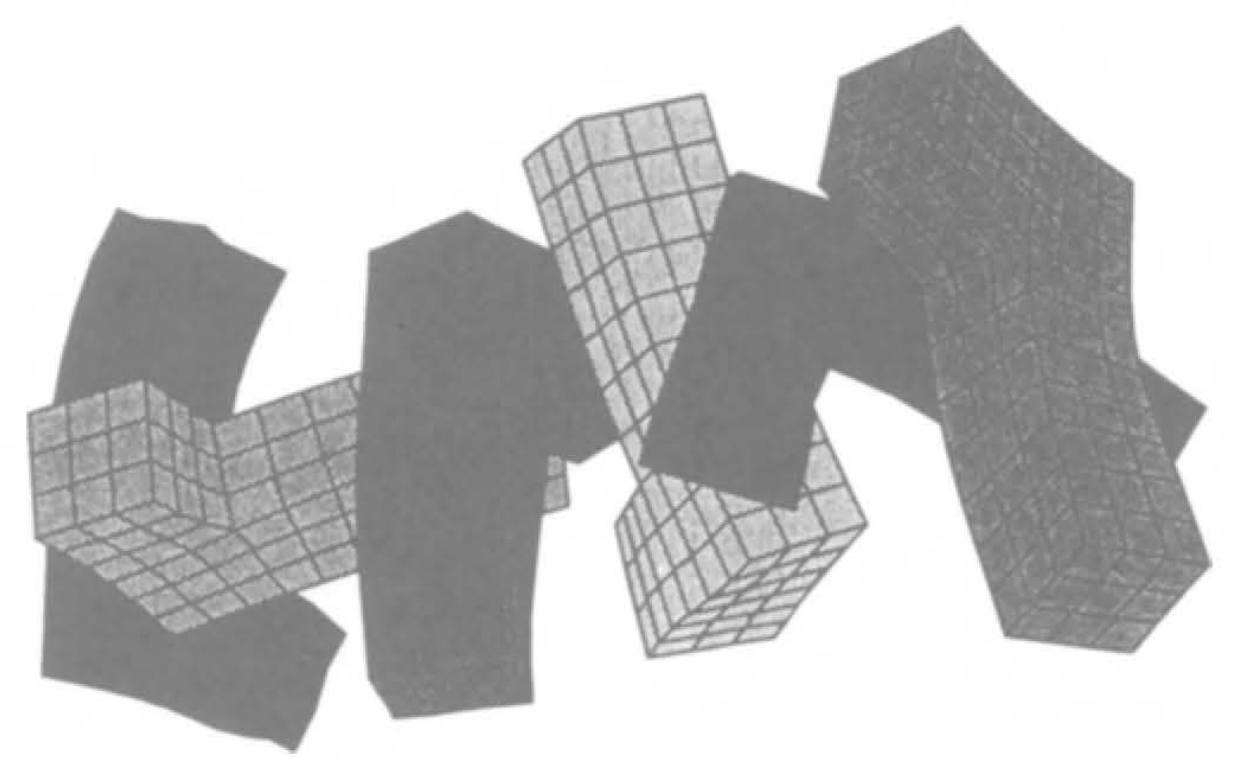

Figure 5.2

Sequence of deformed shapes of the L-shaped block obtained with the exact energy-momentum conserving algorithm for nonlinear elasto-dynamics.

three algorithms, as well as most of the commonly used time stepping schemes, trivially preserve the conservation law of total linear momentum. The results shown in Figure 5.3 include computations with several stepsizes and demonstrate the excellent performance exhibited by the energymomentum algorithm. In particular, the exact conservation of total energy yields an unconditionally stable scheme. In sharp contrast with this performance, both the symplectic mid-point rule and the trapezoidal rule exhibit a significant energy growth for the larger time-step sizes in long-term calculations, which leads to an eventual blow-up of the algorithmic solution and strongly suggests a loss of the unconditional stability property of these methods in the nonlinear regime.

We remark that the exact conservation of total angular momentum exhibited by the mid-point rule is in agreement with the analysis in Simo, Tarnow and Wong [1992]. Although the analysis in this latter reference shows that the trapezoidal rule fails to conserve total angular momentum, the results in Figure 3.3 indicate an excellent overall behavior. We attribute this good performance to the small rotational increments present in this problem as a result of the step-sizes employed. Nevertheless, failure of trapezoidal rule to conserve angular momentum may become significant in other situations.

\subsection{Dynamics of a flexible satellite-like structure}

In the second example we present the simulation of dynamics of a satellite-like structure with constitutive response again modeled by a Saint Venant-Kirchhoff material. Figure 5.4 contains all the necessary data 
Energy-Momentum Method: Energy

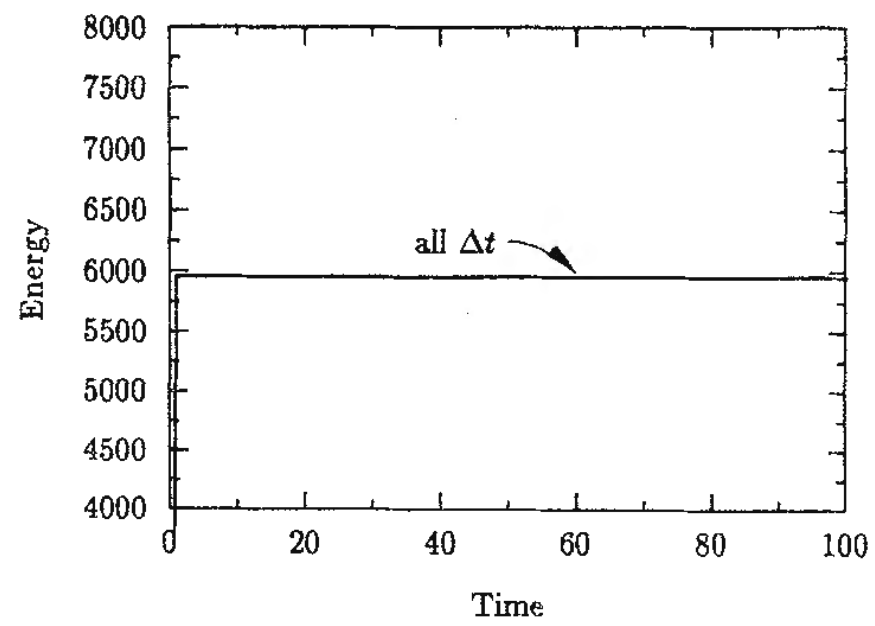

Symplectic Midpoint Rule: Energy

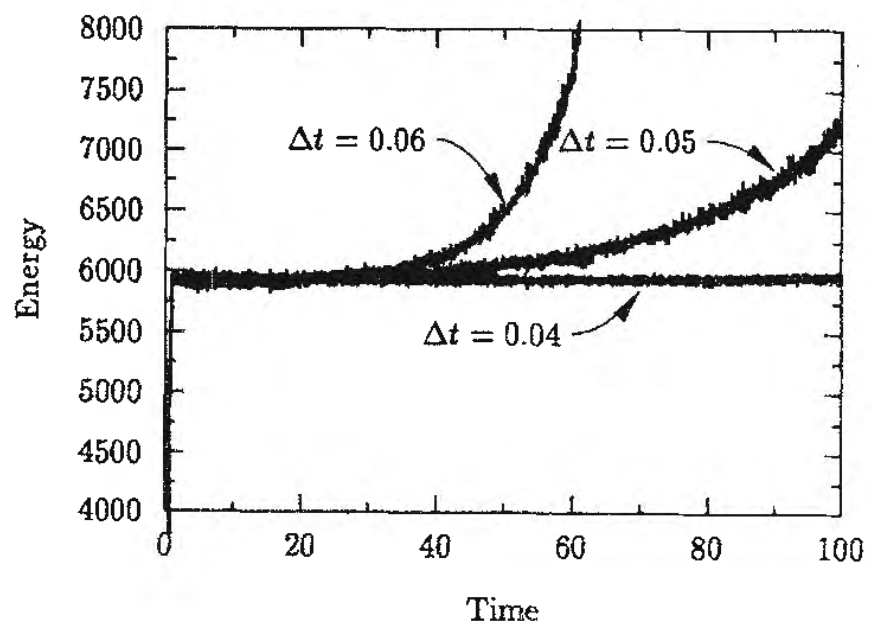

Newmark Method: Energy

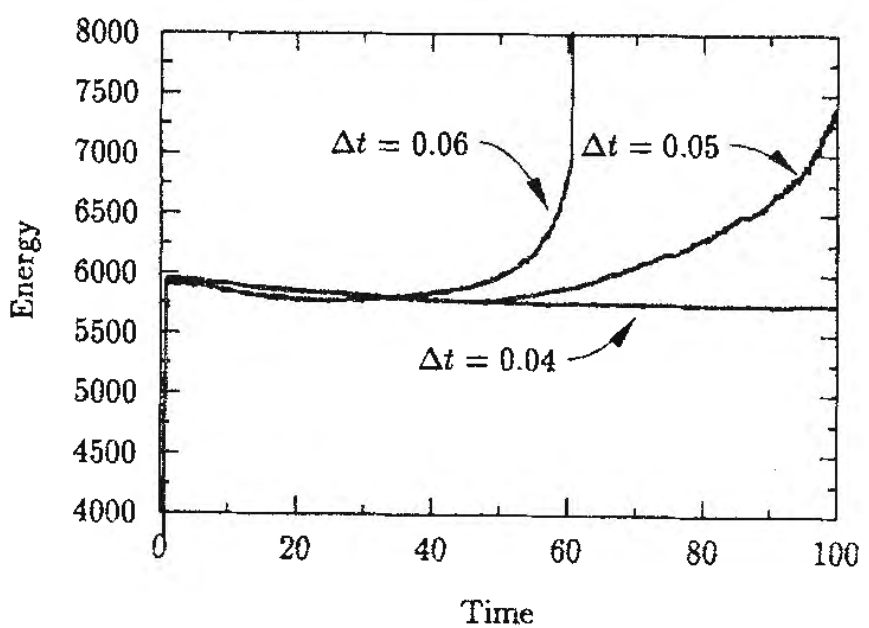

Energy-Momentum Method: Ang. Momentum

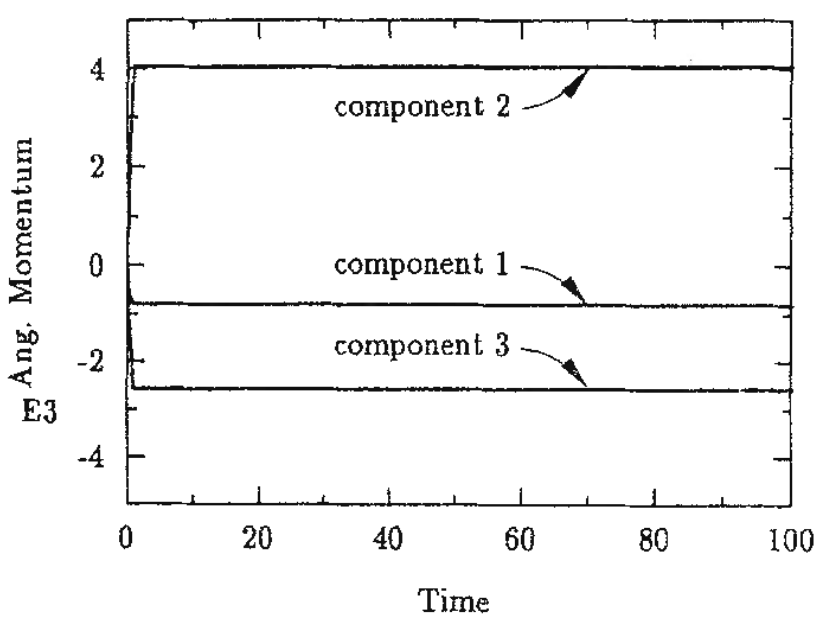

Symplectic Midpoint Rule: Ang. Momentum

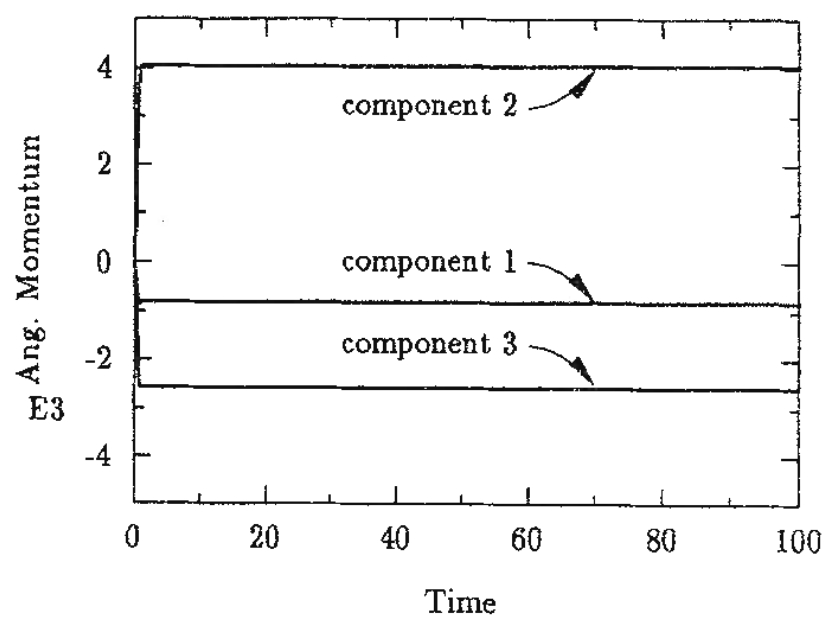

Newmark Method: Ang. Momentum

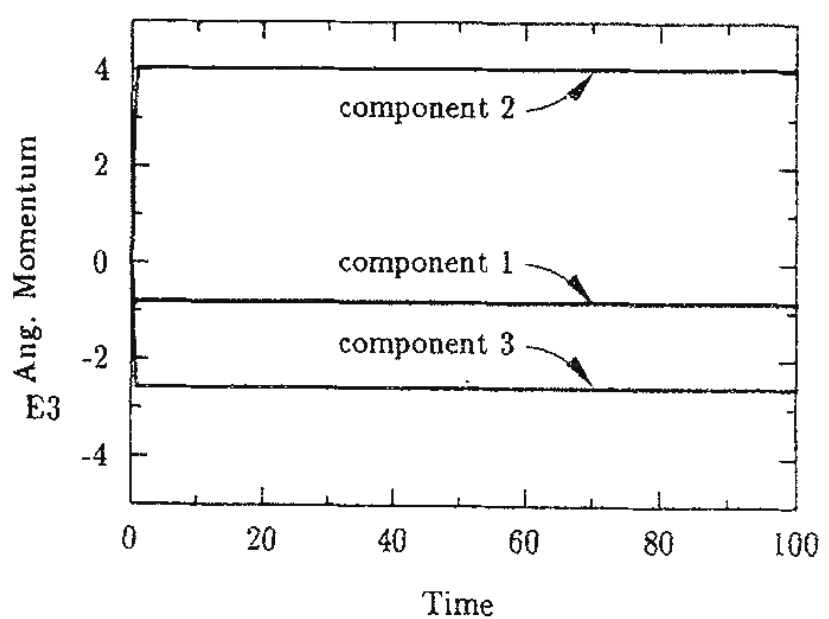

Figure 5.3

Time histories of the total angular momentum and the total energy computed with the exact energymomentum conserving algorithm, the symplectic mid-point rule and the trapezoidal rule.

pertaining to the geometry of the problem, initial conditions and material properties.

As in the preceding example, after an initial loading phase the loads are removed and the subsequent motion of the structure in the ambient space exactly conserves momentum and energy. A representative deformed shape 
$\begin{aligned} & \text { load on the end } \\ & \text { of cantilever A }\end{aligned}=\left\{\begin{array}{l}F_{x}=-400 \cdot p(t) \\ F_{y}=-2000 . \cdot p(t) \\ F_{z}=800 \cdot \cdot p(t)\end{array}\right.$

A

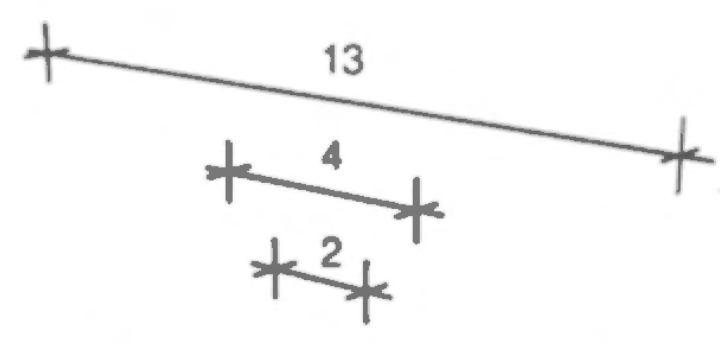

load on the end $=\left\{\begin{array}{l}F_{x}=2000 \cdot \cdot p(t) \\ F_{y}=-400 \cdot \cdot p(t) \\ F_{z}=-200 \cdot \cdot p(t)\end{array}\right.$

load on the end of cantilever C $^{2}=\left\{\begin{array}{l}F_{x}=400 \cdot \cdot p(t) \\ F_{y}=2000 . \cdot p(t) \\ F_{z}=-800 . \cdot p(t)\end{array}\right.$

load on the end $=\left\{\begin{array}{l}F_{x}=-2000 \cdot \cdot p(t) \\ F_{y}=400 \cdot p(t) \\ F_{z}=600 \cdot \cdot p(t)\end{array}\right.$

coad on $=\left\{\begin{array}{l}F_{x}=9600 \cdot p(t) \\ F_{y}=0 . \\ F_{z}=4800 \cdot p(t)\end{array}\right.$

$p(t)=\left\{\begin{aligned} 0.1 \cdot t & \text { for } t \leq 0.15 \\ 0.015-0.1 \cdot t & \text { for } \quad 0.15 \leq t \leq 0.3 \\ 0 . & \text { for } t \geq 1.0\end{aligned}\right.$

Figure 5.4

Geometry and finite element mesh for the simulation of the dynamics of a flexible satelite-like structure, with material response governed by the Saint Venant-Kirchhoff elastic model. The body is initially at rest and then subjected to the loading given in the figure.

Figure 5.5

Finite element mesh and representative deformed shape obtained in the course of the full dynamic response of a flexible satellite-like structure, with material response governed by the Saint VenantKirchhoff elastic model. 
Energy-Momentum Method: Energy
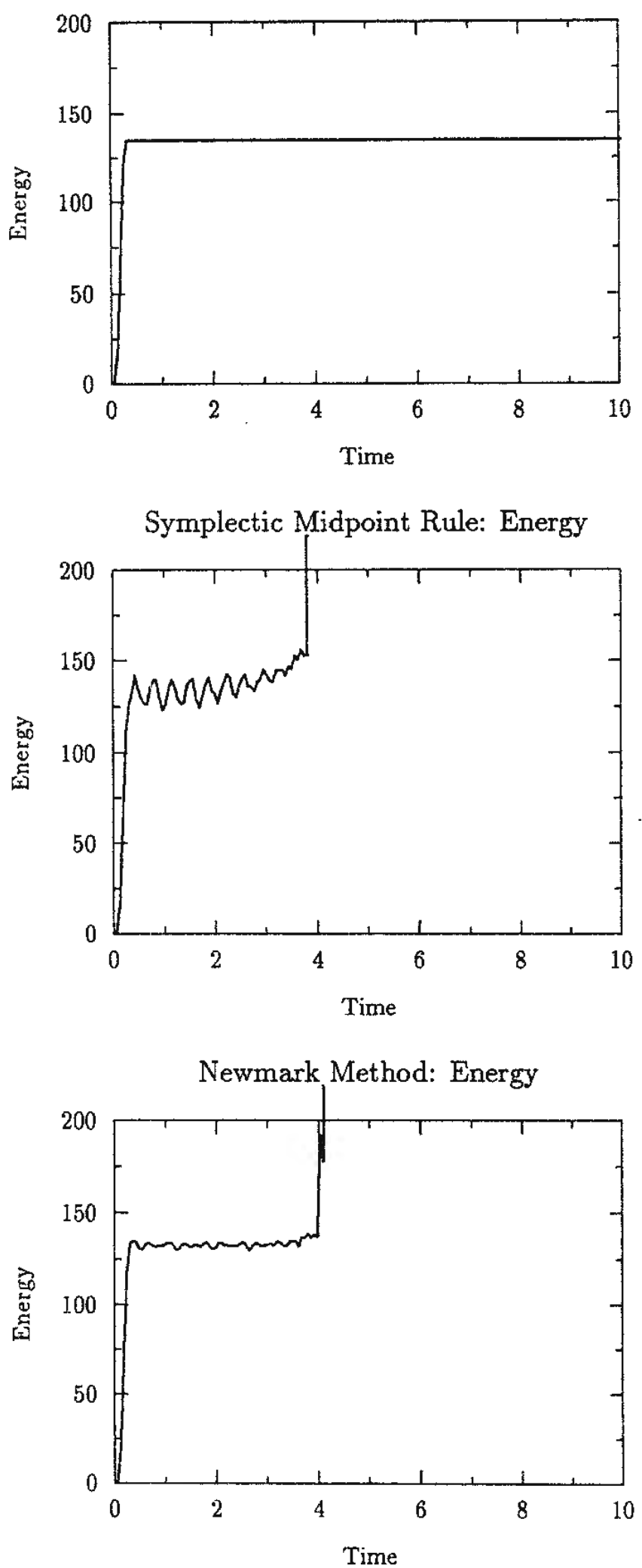

Energy-Momentum Method: Ang. Momentum

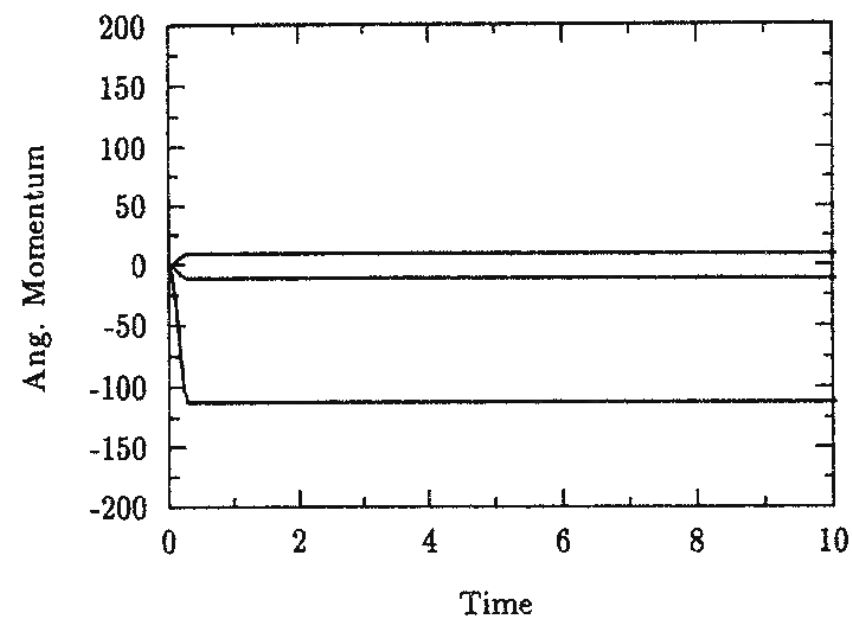

Symplectic Midpoint Rule: Ang. Momentum

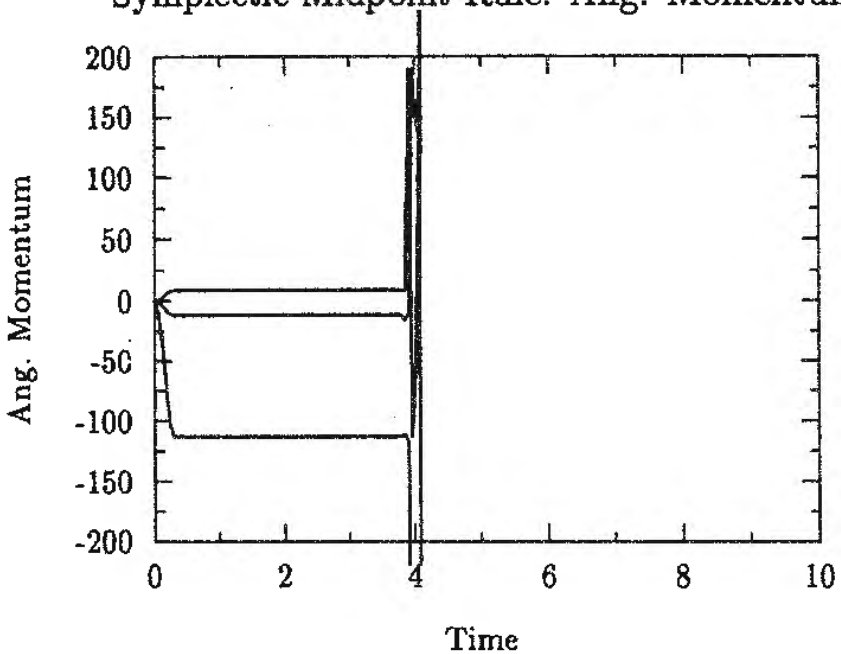

Newmark Method: Ang. Momentum

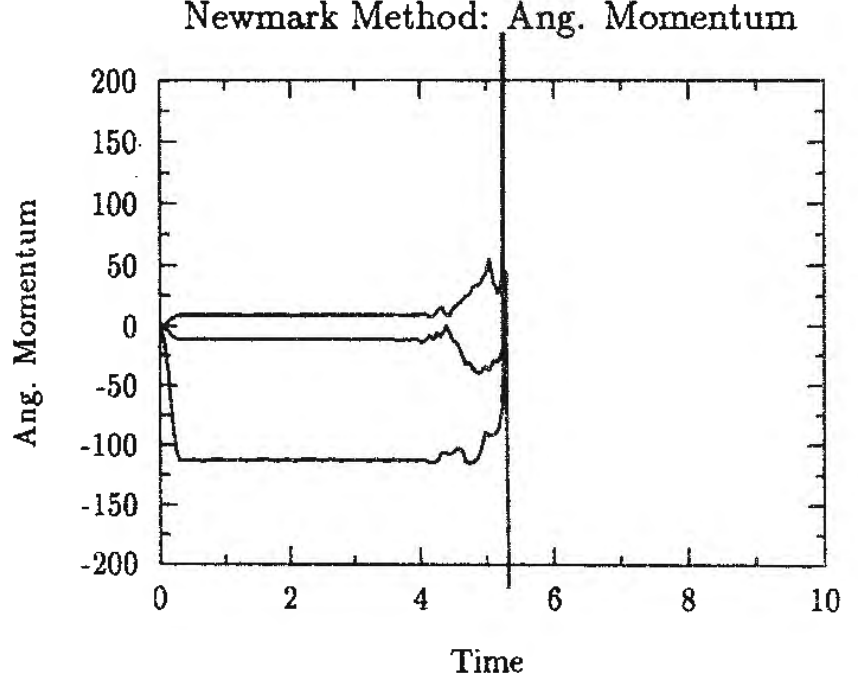

Figure 5.6

Time histories of the total angular momentum and the total energy computed with the exact energymomentum conserving algorithm, the symplectic mid-point rule and the trapezoidal rule.

of the structure is shown in Figure 5.5 without magnification of the actual deformations and demonstrates the presence of finite strains. The results of this numerical simulation are summarized in Figure 5.6 and confirm the conclusions drawn from the first example concerning conservation of momentum and energy exhibited by the three algorithms. Particularly striking 
is the robustness exhibited by the energy-momentum algorithm in comparison to the two other schemes considered, admitting significantly larger time-steps without loss of convergence during the Newton-Raphson iteration.

\section{§6. Summary and conclusions}

We have presented a new methodology for the construction of time stepping algorithms that inherit, by design, the conservation laws of momentum along with an a-priori estimate on the rate of decay of the total energy. For elastodynamics, the case examined in detail in this paper, we have presented a new class of second order accurate, unconditionally stable, exact energy and momentum conserving algorithms. The excellent performance exhibited by this conserving scheme has been demonstrated in two numerical simulations involving true finite strains accompanied by large overall rotations. It has been shown that the proposed method remains stable while two widely used algorithms, the Newmark trapezoidal rule and the symplectic mid-point rule, exhibit blow-up for finite step sizes. Remarkably, the actual implementation of these energy-momentum schemes is essentially identical to the one of standard schemes, and involves negligible additional cost.

The simulations in Section 5 demonstrate how algorithms possessing the same asymptotic order of accuracy and identical properties in the linear regime can exhibit drastically different responses in the nonlinear regime. In this connection, it should be emphasized that the three algorithms compared in Section 5 do become identical when restricted to the linear regime. Although these schemes are unconditionally stable in the linear regimes, their performance in the nonlinear regime is vastly different. The numerical result in Section 5 appear to suggest, therefore, that algorithmic conservation properties play an important role in the practical assessment of the actual performance to be expected from a given algorithm in the nonlinear regime. In particular, conservation of the total energy for a Hamiltonian system such as elastodynamics is not only desirable from a physical standpoint, but also a manifestation of unconditionally (nonlinear) stability of the algorithm.

The ideas set forth herein are by no means restricted to elastodynamics and can be applied, in particular, to dynamical systems exhibiting (positive) internal dissipation. In future publications we plan to exploit this framework in the design of algorithms for dissipative systems such as the Navier-Stokes models. In addition, these ideas can be easily extended to Hamiltonian systems possessing a significantly more complex geometric structure, such as rod and shells. Finally, we plan to address the design of conserving schemes possessing higher order accuracy. 


\section{Acknowledgements}

We are indebted to D. Lewis and $\mathrm{K}$. Wong for helpful comments and many discussions on the subject of this paper. Support for this research was provided by AFOSR under contract No. 2-DJA-826 with Stanford University. This support and the interest and comments of Dr. Marc Jacobs are gratefully acknowledged.

\section{Appendix I. Accuracy analysis of energy-momentum schemes}

In this Appendix we outline the accuracy analysis of the discrete energy-momentum schemes presented in Section 4. Recall that the time discretization of the algorithmic weak form is

$$
\left.\begin{array}{l}
\left\langle\varrho_{0}\left(\boldsymbol{V}_{n+1}-\boldsymbol{V}_{n}\right), \boldsymbol{\eta}\right\rangle+\Delta t\left\langle D \boldsymbol{\varphi}_{n+(1 / 2)} \boldsymbol{S}, \operatorname{grad}[\boldsymbol{\eta}]\right\rangle:=\tau_{\Delta t} \equiv 0, \\
\boldsymbol{\varphi}_{n+1}-\boldsymbol{\varphi}_{n}-\frac{\Delta t}{2}\left[\boldsymbol{V}_{n+1}+\boldsymbol{V}_{n}\right]:=\tau_{\Delta t} \equiv 0,
\end{array}\right\}
$$

where we have assumed for simplicity zero external loading. We restrict our attention to the case in which the algorithmic constitutive equation for $\boldsymbol{S}$ is defined as

$$
\boldsymbol{S}=\nabla \hat{e}\left(\boldsymbol{C}_{n+\beta}\right)+\nabla \hat{e}\left(\boldsymbol{C}_{n+(1-\beta)}\right),
$$

with $\beta \in(0,1)$ defined so that energy is exactly conserved; as explained in Section 4. The analysis of the first order accurate scheme, corresponding to $S=2 \nabla \hat{e}\left(C_{n+\beta}\right)$ follows along identical lines and further details are omitted.

Let $\left(\varphi\left(\cdot, t_{n+1}\right), V\left(\cdot, t_{n+1}\right)\right)$ denote the exact solution at time $t_{n+1}$ defined on $\mathscr{B}$. A Taylor series expansion about $t_{n}$ then yields

$$
\left.\begin{array}{l}
\varphi\left(\cdot, t_{n+1}\right)=\varphi\left(\cdot, t_{n}\right)+\Delta t \dot{\varphi}\left(\cdot, t_{n}\right)+\frac{1}{2} \Delta t \ddot{\varphi}\left(\cdot, t_{n}\right)+\mathcal{O}\left(\Delta t^{3}\right), \\
V\left(\cdot, t_{n+1}\right)=V\left(\cdot, t_{n}\right)+\Delta t \dot{\boldsymbol{V}}\left(\cdot, t_{n}\right)+\frac{1}{2} \Delta t \ddot{\boldsymbol{V}}\left(\cdot, t_{n}\right)+\mathcal{O}\left(\Delta t^{3}\right), \\
D \varphi\left(\cdot, t_{n+(1 / 2)}\right)=D \varphi\left(\cdot, t_{n}\right)+\frac{1}{2} \Delta t D \dot{\varphi}\left(\cdot, t_{n}\right)+\mathcal{O}\left(\Delta t^{2}\right) .
\end{array}\right\}
$$

Setting $\boldsymbol{F}_{n+1}:=D \varphi\left(\cdot, t_{n+1}\right)$ and $\boldsymbol{F}_{n}:=D \varphi\left(\cdot, t_{n}\right)$ a Taylor series expansion of $C_{n+\beta}$ yields

$$
\begin{aligned}
\boldsymbol{C}_{n+\beta} & =\beta \boldsymbol{F}_{n+1}^{T} \boldsymbol{F}_{n+1}+(1-\beta) \boldsymbol{F}_{n}^{T} \boldsymbol{F}_{n} \\
& =\boldsymbol{F}_{n}^{T} \boldsymbol{F}_{n}+\beta \Delta t\left[\dot{\boldsymbol{F}}_{n}^{T} \boldsymbol{F}_{n}+\boldsymbol{F}_{n}^{T} \dot{\boldsymbol{F}}_{n}\right]+\mathcal{O}\left(\Delta t^{2}\right) \\
& =\boldsymbol{C}_{n}+\beta \Delta t \dot{\boldsymbol{C}}_{n}+\mathcal{O}\left(\Delta t^{2}\right) .
\end{aligned}
$$


Inserting this last result into the Taylor series expansion of the algorithmic constitutive equation (I.2) about $t_{n}$ gives the following result

$$
\begin{aligned}
\boldsymbol{S}= & {\left[\nabla \hat{e}\left(\boldsymbol{C}_{n}\right)+\beta \Delta t \nabla^{2} \hat{e}\left(\boldsymbol{C}_{n}\right): \dot{\boldsymbol{C}}_{n}+\mathcal{O}\left(\Delta t^{2}\right)\right.} \\
& \left.+\nabla \hat{e}\left(\boldsymbol{C}_{n}\right)+(1-\beta) \Delta t \nabla^{2} \hat{e}\left(\boldsymbol{C}_{n}\right): \dot{\boldsymbol{C}}_{n}+\mathcal{O}\left(\Delta t^{2}\right)\right] \\
= & 2 \nabla \hat{e}\left(\boldsymbol{C}_{n}\right)+\Delta t \nabla^{2} \hat{e}\left(\boldsymbol{C}_{n}\right): \dot{\boldsymbol{C}}_{n}+\mathcal{O}\left(\Delta t^{2}\right) .
\end{aligned}
$$

Now let $\tau(\Delta t)$ denote the result obtained by inserting the exact solution into the left-hand-side of the algorithmic equation (I.1) ${ }_{1}$. Making use of the expansions (I.5) and (I.3) associated with the exact solution we obtain the following result for (I.1) $)_{1}$, ordered in increasing powers of $\Delta t$ :

$$
\begin{aligned}
\tau(\Delta t):= & \Delta t\left[\left\langle\varrho_{0} \dot{\boldsymbol{V}}_{n}, \boldsymbol{\eta}\right\rangle+\left\langle D \boldsymbol{\varphi}_{n} 2 \nabla \hat{e}\left(\boldsymbol{C}_{n}\right), \operatorname{grad}[\boldsymbol{\eta}]\right\rangle\right] \\
& +\frac{\Delta t^{2}}{2}\left[\left\langle\varrho_{0} \ddot{\boldsymbol{V}}_{n}, \boldsymbol{\eta}\right\rangle+\left\langle D \dot{\boldsymbol{\varphi}}_{n} 2 \nabla \hat{e}\left(\boldsymbol{C}_{n}\right)+2 D \boldsymbol{\varphi}_{n} \nabla^{2} \hat{e}\left(\boldsymbol{C}_{n}\right): \dot{\boldsymbol{C}}_{n}, \operatorname{grad}[\boldsymbol{\eta}]\right\rangle\right] \\
& +\mathcal{O}\left(\Delta t^{3}\right)
\end{aligned}
$$

Similarly, denoting by $\tau(\Delta t)$ the result obtained by inserting the exact solution into the left-hand side of the algorithmic equation $(\mathrm{I} .1)_{2}$ we find

$$
\tau(\Delta t):=\Delta t\left[\dot{\varphi}\left(\cdot, t_{n}\right)-V\left(\cdot, t_{n}\right)\right]+\frac{\Delta t^{2}}{2}\left[\ddot{\varphi}\left(\cdot, t_{n}\right)-\dot{V}\left(\cdot, t_{n}\right)\right]+\mathcal{O}\left(\Delta t^{3}\right) .
$$

By assumption, the weak form of the equations and its time differentiation hold at time $t_{n}$. Consequently, in view of the form of (I.6a,b) it follows that

$$
\tau(\Delta t)=\mathcal{O}\left(\Delta t^{3}\right) \text { and }\|\tau(\Delta t)\|=\mathcal{O}\left(\Delta t^{3}\right) .
$$

Recalling the classical definition of the local truncation error (see e.g., Richtmyer and Morton [1967]) we conclude that the proposed energymomentum algorithm is second order accurate for any value $\beta \in[0,1]$.

\section{Appendix II. Symplectic integration of Hamiltonian systems}

This Appendix summarizes the Hamiltonian structure underlying Galerkin finite element discretizations on nonlinear elastodynamics. The main goal is to investigate to what extent the proposed discrete energymomentum conserving algorithm defines a symplectic scheme. Recall that an algorithm is called symplectic if the (generally implicit) map that advances the solution in phase space from time $t_{n}$ to time $t_{n+1}$ defines a symplectic transformation. This type of algorithms has received a considerable amount of attention in recent years; see e.g., Ruth [1983], Channell [1983], Lasagni [1988], Sanz-Serna [1988] and the recent review paper of Scovel [1991]. The underlying motivation is the belief, to some extent 
unsubstantiated, that preserving the symplectic character of the Hamiltonian flow will enhance the long-term characteristics of an algorithm. In contrast with the notions of energy and momentum, which remain applicable to dissipative systems, the symplectic property is clearly restricted to Hamiltonian system.

If the Hamiltonian system is non-integrable, a result of Zhong and Marsden [1988] implies that an energy preserving algorithm which is also symplectic can differ from the exact Hamiltonian flow only up to a time reparametrization and, therefore, it is unlikely to exist. Since nonlinear elastodynamics is a non-integrable system, the conclusions of the analysis given below are consistent with this observation. We show that the energymomentum conserving scheme defines a symplectic transformation only up to terms of order $\Delta t^{3}$. Additionally, we show that the mid-point rule is the only member of the one parameter family of generalized mid-point rule schemes which is symplectic. We remark that our numerical simulations in Section 5 strongly suggest that exact momentum and energy conservation result in significantly better performance in long-term dynamic calculations than exact conservation of the symplectic structure.

\section{II.1. Discrete canonical Hamiltonian structure}

We outline below the Hamiltonian structure of the finite dimensional dynamical system obtained via a Galerkin discretization on nonlinear elastodynamics, as described in Section 4. This Hamiltonian structure is considered in its own right, with independence of the Hamiltonian structure of the infinite dimensional dynamical system, as described in Simo, Marsden and Krishnaprassad [1988], which plays no role in these developments. Accordingly, given the discrete state space $\mathscr{S}^{h}$ described in Section 4, define

$$
\boldsymbol{p}^{A}:=\sum_{B=1}^{n_{\text {node }}} M^{A B} V_{B} \quad \text { with } \quad M^{A B}:=\int_{\mathscr{B}} \varrho_{0} N^{A}(X) N^{B}(X) d \mathscr{B}
$$

and introduce the following vector notation

$$
\begin{aligned}
& \boldsymbol{q}:=\left(\boldsymbol{d}_{1}^{\prime}, \boldsymbol{d}_{2}, \ldots, \boldsymbol{d}_{n_{\text {node }}}\right) \in \mathbb{R}^{n_{\text {dof }}}, \\
& \boldsymbol{p}:=\left(\boldsymbol{p}^{1}, \boldsymbol{p}^{2}, \ldots, \boldsymbol{p}^{n_{\text {node }}}\right) \in \mathbb{R}^{n_{\text {dor }}},
\end{aligned} \text { and } \boldsymbol{z}:=\left\{\begin{array}{l}
\boldsymbol{q} \\
\boldsymbol{p}
\end{array}\right\} .
$$

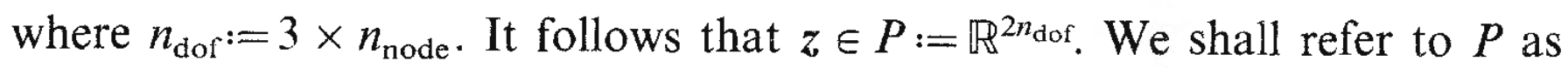
the nodal phase space associated with Galerkin finite element discretizations of nonlinear elastodynamics. On $P$ one defines the kinetic energy function via the expression

$$
K(\boldsymbol{p}):=\frac{1}{2} \boldsymbol{p} \cdot \boldsymbol{M}^{-1} \boldsymbol{p}>0, \quad \text { with } \quad \boldsymbol{M}=\left[\boldsymbol{M}^{A B} \mathbf{1}_{3 \times 3}\right] \in \mathbb{R}^{n_{\mathrm{dof}}} \times \mathbb{R}^{n_{\mathrm{dof}}} .
$$

Next, one defines the potential energy function $U: \mathbb{R}^{n_{\mathrm{dof}}} \rightarrow \mathbb{R}$ as follows. Recall that the deformation gradient $D \varphi^{h}$ and the right Cauchy-Green 
tensor $C\left(\varphi^{h}\right)$ associated with a configuration $\varphi^{h}=\sum_{A=1}^{n_{\text {node }}} N^{A} d_{A}$ are given by

$$
D \varphi^{h}=\sum_{A=1}^{n_{\text {node }}} d_{A} \otimes \operatorname{grad}\left[N^{A}(X)\right] \text { and } C\left(\varphi^{h}\right):=\left[D \varphi^{h}\right]^{T} D \varphi^{h} .
$$

Using if necessary a numerical quadrature formula with total number of quadrature points $n_{\text {quad }}$ weights $g_{l}, l=1, \ldots, n_{\text {quad }}$ the potential energy function becomes

$$
U(\boldsymbol{q}):=\int_{\mathscr{B}} \hat{e}\left(\boldsymbol{C}\left(\boldsymbol{\varphi}^{h}\right)\right) d \mathscr{B}=\sum_{l=1}^{n_{\text {quad }}} \hat{e}\left(\boldsymbol{C}\left(\boldsymbol{\varphi}_{l}^{h}\right)\right) \boldsymbol{g}_{l},
$$

where $\varphi_{l}^{h}$ denotes the configuration $\varphi^{h}$ evaluated at the quadrature point $X_{l}$.

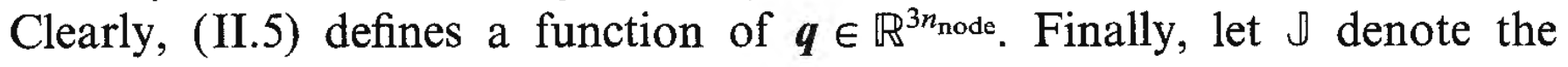
symplectic $n_{\text {dof }} \times n_{\text {dof }}$ defined by

$$
\mathbb{J}:=\left[\begin{array}{rr}
0 & 1 \\
-1 & 0
\end{array}\right] \text {, }
$$

where $\mathbf{0}$ and $\mathbf{1}$ are the zero and unit $n_{\text {dof }} \times n_{\text {dof }}$ matrices, respectively. Recall that $₫$ possess the following well-known properties

$$
\rrbracket=-\rrbracket^{T}=-\rrbracket^{-1} \text { and } \operatorname{det}[\rrbracket]=1 .
$$

With the foregoing notation in hand, the Galerkin discretization of nonlinear elastodynamics possess a canonical Hamiltonian structure with

i. (Nodal) canonical phase space $P=\mathbb{R}^{2 n_{\text {dof }}}$

ii. (Separable) Hamiltonian $H(z)=K(p)+U(q)$

iii. (Canonical) symplectic two-form $w\left(\delta \boldsymbol{z}_{1}, \delta \boldsymbol{z}_{2}\right):=\delta \boldsymbol{z}_{1}^{T} \rrbracket \delta \boldsymbol{z}_{2}$, for $\delta \boldsymbol{z}_{1}$, $\delta z_{2} \in T_{z} P$.

Here $T_{z} P$ denotes the tangent space at $z \in P$ of admissible variations. For such a Hamiltonian system, the canonical equations of motion take the standard form

$$
\left.\begin{array}{l}
\frac{d}{d t}(z(t))=\nabla \nabla H(z(t)) \\
\left.z(t)\right|_{t=0}=z_{0}
\end{array}\right\} \quad \text { with } \nabla H(z):=\left\{\begin{array}{l}
\nabla U(q) \\
\boldsymbol{M}^{-1} p
\end{array}\right\} .
$$

We shall denote by $\mathbb{F}_{t}: P \rightarrow P$ the flow generated by equations (II.8), defined by the standard relation $z(t)=\mathbb{F}_{t}\left(z_{0}\right)$.

\section{II.2. Symplectic algorithms}

Recall that the symplectic two-form defined above is conserved by the Hamiltonian flow $\mathbb{F}_{t}(\cdot)$ in the sense that the relation

$$
\frac{d}{d t} w\left(D \mathbb{F}_{t}(z) \delta z_{1}, D \mathbb{F}_{t}(z) \delta z_{2}\right)=0,
$$


holds for arbitrary variations $\delta z_{1}, \delta z_{2} \in P$. Here $D \mathbb{F}_{t}(z)$ denotes the derivative of $\mathbb{F}_{t}$ at $z \in P$ defined in canonical coordinates by the $2 n_{\text {dof }} \times 2 n_{\text {dof }}$ matrix of partial derivatives. Condition (II.9) is equivalent to the requirement

$$
\left[D \mathbb{F}_{t}(z)\right]^{T} \Downarrow D \mathbb{F}_{t}(z)=\mathbb{d} ;
$$

in other words, the matrix $D \mathbb{F}_{t}(z)$ is symplectic. Condition (II.9) has a number of well-known consequences. In particular, from (II.10) one concludes that the Hamiltonian flow preserves the volume in phase space (Liouville's theorem) since $\operatorname{det}\left[D \mathbb{F}_{t}(\boldsymbol{z})\right]=1$.

In general, an arbitrary mapping $\mathbb{G}: P \rightarrow P$ which satisfies condition (II.9) [or (II.10) with $\mathbb{F}_{t}$ replaced by $\mathbb{G}$ ] is called a symplectic transformation. Symplectic maps are canonical transformations which preserve the form of Hamilton's equations, the volume in phase space and the integral invariants. In particular, an algorithm defines a time-one-map in phase space which advances the initial conditions $z_{n} \in P$ at time $t_{n}$ to the algorithmic solution $z_{n+1} \in P$ according to a generally implicit rule $\mathbb{G}\left(z_{n+1}, z_{n}\right)=\mathbf{0}$ consistent with Hamilton's equations. The algorithm is thus said to be symplectic if this time-one-map is a symplectic transformation. A direct verification of this condition involves considering the linearized algorithm $\delta z_{n+1}=\boldsymbol{A} \delta \boldsymbol{z}_{n}$. The matrix $\boldsymbol{A}$ is called the linearized amplification matrix of the scheme and is given by

$$
\boldsymbol{A}=\boldsymbol{A}_{1}^{-1} \boldsymbol{A}_{2}
$$

where

$$
\boldsymbol{A}_{1}:=\left[\frac{\partial \mathbb{G}\left(\boldsymbol{z}_{n+1}, \boldsymbol{z}_{n}\right)}{\partial \boldsymbol{z}_{n+1}}\right] \text { and } \boldsymbol{A}_{2}:=-\left[\frac{\partial \mathbb{G}\left(\boldsymbol{z}_{n+1}, \boldsymbol{z}_{n}\right)}{\partial \boldsymbol{z}_{n}}\right] \text {. }
$$

It follows that the time-stepping scheme is symplectic if $\boldsymbol{A}^{T} \rrbracket \boldsymbol{A}=\mathbb{}$. From a computational stand point, an alternative form of this condition that avoids inversion of the matrix $\boldsymbol{A}_{1}$, and hence much easier to check in applications, is derived as follows. From (II.11) we conclude that

$$
A^{T} \rrbracket A=\rrbracket \Leftrightarrow\left[A_{1} \rrbracket^{-1} A_{1}^{T}\right]^{-1}=\left[A_{2} \rrbracket^{-1} A_{2}^{T}\right]^{-1},
$$

and using properties (II.6) we arrive at the equivalent characterization

$$
\boldsymbol{A}^{T} \rrbracket \boldsymbol{A}=\rrbracket \Leftrightarrow \boldsymbol{A}_{1} \rrbracket \boldsymbol{A}_{1}^{T}-\boldsymbol{A}_{2} \rrbracket \boldsymbol{A}_{2}^{T}=\mathbf{0} .
$$

We remark that the spectral stability properties of the algorithm can be inferred from the roots of the characteristic polynomial $p(\lambda):=\operatorname{det}\left[\boldsymbol{A}-\lambda \boldsymbol{I}_{2 n}\right]$. Since $\operatorname{det}[A]=1$ one concludes that the spectral radius $\varrho$ of symplectic integrators must be $\varrho=1$. As a result, a spectrally stable algorithm which exhibits numerical dissipation cannot be symplectic. 
II.3. Approximate symplectic character of the energy-momentum scheme

First, we compute the amplification matrix of the one-parameter family of generalized mid-point rule algorithms and use condition (II.12) to show that the mid-point rule is the only symplectic member of this family. This result appears to be not well-known although the symplectic character of the mid-point rule is well-established, see Feng Kan [1986]. Subsequently, we exploit this result in an expansion of the discrete energy-momentum algorithm in powers of the time-step $\Delta t$ to conclude that this latter scheme fails to be symplectic, at worst, to order $(\Delta t)^{3}$.

\section{II.3.1. The symplectic midpoint rule}

For a general canonical Hamiltonian system, the one-parameter family of generalized mid-point rule algorithms takes the form

$$
\left.\begin{array}{r}
\mathbb{G}\left(z_{n+1}, z_{n}\right):=z_{n+1}-z_{n}-\Delta t \rrbracket \nabla H\left(z_{n+\alpha}\right)=\mathbf{0}, \\
\boldsymbol{z}_{n+\alpha}=\alpha z_{n+1}+(1-\alpha) z_{n} \quad \text { for } \alpha \in[0,1] .
\end{array}\right\}
$$

Differentiating expression (II.13) $)_{1}$ and using the notation in (II.11) gives the result

$$
\left.\begin{array}{l}
\boldsymbol{A}_{1}=\mathbf{1}-\alpha \Delta t \rrbracket \nabla^{2} H\left(\boldsymbol{z}_{n+\alpha}\right) \\
\boldsymbol{A}_{2}=\mathbf{1}+(1-\alpha) \Delta t \rrbracket \nabla^{2} H\left(\boldsymbol{z}_{n+\alpha}\right)
\end{array}\right\}
$$

With these expressions in hand, condition (II.12) then becomes

$$
\boldsymbol{A}_{1} \rrbracket \boldsymbol{A}_{1}^{T}-\boldsymbol{A}_{2} \rrbracket \boldsymbol{A}_{2}^{T}=(2 \alpha-1) \Delta t^{2}\left[\rrbracket \nabla^{2} H\left(z_{n+\alpha}\right) \unlhd \nabla^{2} H\left(\boldsymbol{z}_{n+\alpha}\right) \rrbracket^{T}\right],
$$

which shows that, in general, the class of algorithm (II.13) is symplectic only if $\alpha=\frac{1}{2}$.

For the Galerkin finite element discretization of nonlinear elastodynamics in Section $4, \nabla H\left(z_{n+\alpha}\right)$ is computed from (II.8) $)_{2}$ evaluated at $z_{n+\alpha}$ with

$$
\left.\begin{array}{l}
\partial_{\boldsymbol{q}_{A}} U(\boldsymbol{q})=\int_{\mathscr{B}} D \boldsymbol{\varphi}_{n+(1 / 2)}^{h} \boldsymbol{S}_{\mathrm{mp}}^{h} \operatorname{grad}\left[N^{A}(X)\right] d \mathscr{B}, \\
\boldsymbol{S}_{\mathrm{mp}}^{h}:=2 \nabla \hat{e}\left(C\left(\boldsymbol{\varphi}_{n+(1 / 2)}^{h}\right)\right),
\end{array}\right\}
$$

where $\varphi_{n+(1 / 2)}^{h}:=\frac{1}{2} \sum_{A=1}^{n_{\text {node }}} N^{A}(X)\left(d_{n A}+d_{n+1 A}\right)$ is the mid-point configuration and $C\left(\varphi_{n+(1 / 2)}^{h}\right)$ is the associated right Cauchy-Green tensor defined by $(\text { II. } 4)_{2}$. 
II.3.2. The discrete energy-momentum method

The algorithmic equations that define the exact energy-momentum conserving algorithm now take the following form

$$
\mathbb{G}\left(z_{n+1}, z_{n}\right):=z_{n+1}-z_{n}-\Delta t \rrbracket\left\{\begin{array}{c}
\boldsymbol{F}_{n+(1 / 2)}\left(\boldsymbol{q}_{n}, \boldsymbol{q}_{n+1}\right) \\
\boldsymbol{M}^{-1} \boldsymbol{p}_{n+(1 / 2)}
\end{array}\right\}=\mathbf{0} .
$$

Thus, in contrast with the symplectic mid-point rule which is defined by (II.13) with $\alpha=\frac{1}{2}$ along with (II.15), the discrete energy-momentum method uses $\boldsymbol{F}_{n+(1 / 2)}^{\text {int }}$ in place of $\nabla U(\boldsymbol{q})$. We recall the expression

$$
\left.\begin{array}{l}
F_{n+(1 / 2)}^{\mathrm{int} A}:=\int_{\mathscr{B}} D \varphi_{n+(1 / 2)}^{h} S_{\mathrm{em}}^{h} \operatorname{grad}\left[N^{A}(X)\right] d \mathscr{B}, \\
\boldsymbol{S}_{\mathrm{em}}^{h}:=\nabla \hat{e}\left(C_{n+\beta}^{h}\right)+\nabla \hat{e}\left(C_{n+(1-\beta)}^{h}\right), \\
C_{n+\alpha}^{h}:=\alpha C\left(\varphi_{n+1}^{h}\right)+(1-\alpha) C\left(\varphi_{n}^{h}\right), \quad \forall \alpha \in[0,1] .
\end{array}\right\}
$$

By comparing (II.18) with (II.15) we conclude that difference between the two methods lies precisely in the definition of the convected stresses; i.e. $S_{\mathrm{em}}^{h}$ versus $S_{\mathrm{mp}}^{h}$. A straightforward Taylor series expansion then gives the relation

$$
\boldsymbol{S}_{\mathrm{em}}^{h}=\boldsymbol{S}_{\mathrm{mp}}^{h}+\mathcal{O}\left(\Delta t^{2}\right) \Rightarrow \boldsymbol{F}_{n+(1 / 2)}^{\mathrm{int}}=\nabla U\left(\boldsymbol{q}_{n+(1 / 2)}\right)+\mathcal{O}\left(\Delta t^{2}\right) .
$$

It therefore follows that the matrices $\boldsymbol{A}_{1}^{\mathrm{em}}$ and $\boldsymbol{A}_{2}^{\mathrm{em}}$ associated with the energy-momentum method and the corresponding matrices for the symplectic mid-point rule are related by the estimates

$$
\boldsymbol{A}_{1}^{\mathrm{em}}=\boldsymbol{A}_{1}^{\mathrm{mp}}+\left[\mathcal{O}\left(\Delta t^{3}\right)\right] \quad \text { and } \quad \boldsymbol{A}_{2}^{\mathrm{em}}=\boldsymbol{A}_{2}^{\mathrm{mp}}+\left[\mathcal{O}\left(\Delta t^{3}\right)\right] .
$$

As a result, we obtained the following estimate for condition (II.12):

$$
\boldsymbol{A}_{1}^{\mathrm{em}} \rrbracket\left[\boldsymbol{A}_{1}^{\mathrm{em}}\right]^{T}-\boldsymbol{A}_{2}^{\mathrm{em}} \rrbracket\left[\boldsymbol{A}_{2}^{\mathrm{em}}\right]^{T}=\left[\mathcal{O}\left(\Delta t^{3}\right)\right],
$$

which shows that the discrete energy-momentum method defines a symplectic transformation up to terms of order $\mathcal{O}\left(\Delta t^{3}\right)$.

\section{References}

V. I. Arnold, Mathematical Methods of Classical Mechanics, Springer Verleg, New York 1988.

A. Bayliss and E. Isaacson, How to make your algorithm conservative, American Math. Soc., A594-A595 (1975).

P. J. Channell, Symplectic Integration Algorithms, Los Alamos Natl Lab. Int. Rept. AT-6:ATN-83-9 (1983).

P. G. Ciarlet, Mathematical Elasticity Volume I. The Three Dimensional Theory, North-Holland, Amsterdam 1988.

R. de Vogelaere, Methods of integration which preserve the contact transformation property of Hamiltonian equations, Dept. of Math., University of Notre Dame, Rept. 4 (1956).

T. J. R. Hughes, W. K. Liu and P. Caughy, Transient finite element formulations that preserve energy, J. Appl. Mech., 45, 366-370 (1978). 
Feng Kan, Difference schemes for Hamiltonian formalism and symplectic geometry, J. Comp. Math., 4, 279-289 (1986).

J. K. Knowles, On the dissipation associated with equilibrium shocks in finite elasticity, J. Elasticity, 9(2), $131-158$ (1979).

R. A. Labudde and D. Greenspan, Energy and momentum conserving methods of arbitrary order for the numerical integration of equations of motion. Part I, Num. Math., 25, 323-346 (1976a).

R. A. Labudde and D. Greenspan, Energy and momentum conserving methods of arbitrary order for the numerical integration of equations of motion. Part II, Num. Math., 26, 1-16 (1976b).

F. M. Lasagni, Canonical Runge-Kutta methods, ZAMP, 39, 952-953 (1988).

J. E. Marsden, Elementary Classical Analysis, W.H. Freeman and Co., San Francisco 1974.

R. D. Ritchmyer and K. W. Morton, Difference Methods for Initial Value Problems, Interscience, New York, 2nd ed. 1967.

R. D. Ruth, A canonical integration technique, IEEE Trans. Nucl. Sci., 30(4), 2669-2671 (1983).

J. M. Sanz-Serna, Runge-Kutta schemes for Hamiltonian systems, BIT, 28, 877-883 (1988).

C. Scovel, Symplectic numerical integration of Hamiltonian Systems, in The Geometry of Hamiltonian Systems, Proc. Workshop held June 5th to 15th, 1989, pp. 463-496, Tudor Ratiu Ed., Springer-Verlag, Berlin 1991.

J. C. Simo, J. E. Marsden and P. S. Krishnaprasad, The Hamiltonian structure of nonlinear elasticity: The convected representation of solids, rods and plates, Arch. Rat. Mech. Analysis, 104, 125-183 (1989).

J. C. Simo and K. K. Wong, Unconditionally stable algorithms for rigid body dynamics that exactly preserve energy and angular momentum, Int. J. Num. Meth. Eng., 31, 19-52 (1991).

J. C. Simo, Nonlinear stability of the time discrete variational problem in nonlinear heat conduction plasticity and viscoplasticity, Comp. Meth. Appl. Mech. Eng., 88, 111-131 (1991).

J. C. Simo, M. S. Rifai and D. D. Fox, On a stress resultant geometrically shell model. Part VI: Conserving algorithms for nonlinear dynamics, Comp. Meth. Appl. Mech. Eng., 34, 117-164 (1992).

J. C. Simo, N. Tarnow and K. Wong, Exact energy-momentum conserving algorithms and symplectic schemes for nonlinear dynamics, Comp. Meth. Appl. Mech. Eng. (in press).

C. Truesdell and W. Noll, The Nonlinear Field Theories of Mechanics, Handbuch der Physik, Vol. III/3, S. Flügge, Ed., Springer-Verlag, Berlin 1972.

G. Zhong and J. E. Marsden, Lie-Poisson Hamilton-Jacobi theory and Lie-Poisson integrators, Phys. Lett. A, 33(3), 134-139 (1988).

\begin{abstract}
In the absence of external loads or in the presence of symmetries (i.e., translational and rotational invariance) the nonlinear dynamics of continuum systems preserves the total linear and the total angular momentum. Furthermore, under assumption met by all classical models, the internal dissipation in the system is non-negative. The goal of this work is the systematic design of conserving algorithms that preserve exactly the conservation laws of momentum and inherit the property of positive dissipation for any step-size. In particular, within the specific context of elastodynamics, a second order accurate algorithm is presented that exhibits exact conservation of both total (linear and angular) momentum and total energy. This scheme is shown to be amenable to a completely straightforward (Galerkin) finite element implementation and ideally suited for long-term/large-scale simulations. The excellent performance of the method relative to conventional time-integrators is conclusively demonstrated in numerical simulations exhibiting large strains coupled with a large overall rigid motion.
\end{abstract}

